\title{
Myo-Cortical Crossed Feedback Reorganizes Primate Motor Cortex Output
}

\author{
Timothy H. Lucas ${ }^{1}$ and Eberhard E. Fetz ${ }^{2}$ \\ ${ }^{1}$ Department of Neurosurgery, Perelman School of Medicine, University of Pennsylvania, Philadelphia, Pennsylvania 19104 and ${ }^{2}$ Department of Physiology \\ and Biophysics, University of Washington, Seattle, Washington 98195
}

\begin{abstract}
The motor system is capable of adapting to changed conditions such as amputations or lesions by reorganizing cortical representations of peripheral musculature. To investigate the underlying mechanisms we induced targeted reorganization of motor output effects by establishing an artificial recurrent connection between a forelimb muscle and an unrelated site in the primary motor cortex (M1) of macaques. A head-fixed computer transformed forelimb electromyographic activity into proportional subthreshold intracortical microstimulation (ICMS) during hours of unrestrained volitional behavior. This conditioning paradigm stimulated the cortical site for a particular muscle in proportion to activation of another muscle and induced robust site- and input-specific reorganization of M1 output effects. Reorganization was observed within $25 \mathrm{~min}$ and could be maintained with intermittent conditioning for successive days. Control stimulation that was independent of muscle activity, termed "pseudoconditioning," failed to produce reorganization. Preconditioning output effects were gradually restored during volitional behaviors following the end of conditioning. The ease of changing the relationship between cortical sites and associated muscle responses suggests that under normal conditions these relations are maintained through physiological feedback loops. These findings demonstrate that motor cortex outputs may be reorganized in a targeted and sustainable manner through artificial afferent feedback triggered from controllable and readily recorded muscle activity. Such cortical reorganization has implications for therapeutic treatment of neurological injuries.
\end{abstract}

\section{Introduction}

Under normal behavioral conditions, primary motor cortex (M1) sites have a relatively stable bidirectional relationship with limb muscles: stimulating a cortical efferent zone evokes consistent muscle responses (Asanuma and Rosén, 1972) and stimulating muscle receptors activates neurons in the corresponding cortical zones (Rosén and Asanuma, 1972; Cheney and Fetz, 1984). The stability of this reciprocal myo-cortical relationship seems remarkable given abundant evidence that plastic changes of M1 movement representations can be induced by altered circumstances (Sanes and Donoghue, 2000).

These consistent reciprocal relations within myo-cortical loops are normally maintained by the balance of synaptic inputs provided through physiological pathways. However, altered circumstances such as lesions can perturb the feedback conditions and change the activation patterns of neuronal circuits. Consistent changes in the activation of these circuits can induce long-

Received 0ct. 3, 2012; revised Jan. 18, 2013; accepted Feb. 7, 2013.

Author contributions: T.H.L. and E.E.F. designed research; T.H.L. and E.E.F. performed research; T.H.L. and E.E.F. analyzed data; T.H.L. and E.E.F. wrote the paper.

Experiments were funded by the National Institutes of Health (NS12542, RR00166, E.E.F.; NS007144, T.H.L.), the Washington Life Science Discovery Fund (E.E.F.), Washington Royalty Technology Transfer Fund (E.E.F. and T.H.L.), and the Congress of Neurological Surgeons Fellowship in Advanced Neurosystems Modulation (T.H.L.). We thankS Perlmutter, A. Jackson, and C. Moritz for critical discussions relating to the experiments; L. Shupe for programming assistance; G. Wu and Y. Nishimura for technical assistance; and G.A. Ojemann for support.

The authors declare no competing financial interests.

Correspondence should be addressed to Timothy H. Lucas, 3400 Spruce Street, 3rd Floor, Silverstein, Hospital of the University of Pennsylvania, Philadelphia, PA 19104. E-mail: Timothy.Lucas@uphs.upenn.edu.

DOI:10.1523/JNEUROSCI.4683-12.2013

Copyright $\odot 2013$ the authors $\quad 0270-6474 / 13 / 335261-14 \$ 15.00 / 0$ term changes in synaptic strength, and such plasticity is thought to underlie cortical reorganization (Hebb, 1949; Markram et al., 1997; Cramer et al., 2011).

Examples of altered circumstances include the disruption of normal feedback pathways. Cortical reorganization can be produced by selective disruption of pure motor nerves (Sanes et al., 1988), selective deafferentation (Kaas et al., 1983; Pons et al., 1991; Elbert et al., 1994), partial disruption of both motor and sensory pathways (Freund et al., 2011), central lesions (Nudo and Milliken, 1996), and complete loss of bidirectional communication following amputation (Qi et al., 2000) or nerve division (Donoghue and Sanes, 1987).

Similar mechanisms are thought to underlie use-dependent plasticity during normal learning. Acquisition of new motor skills induces expansion of motor representations (Jenkins et al., 1990a; Pascual-Leone et al., 1995; Nudo et al., 1996; Hikosaka et al., 2002), just as sustained practice of sensory discrimination expands sensory representations (Jenkins et al., 1990b; Recanzone et al., 1992a,b). Representational expansion precedes the explicit phase of learning a new skill, demonstrating that rapid functional plasticity of cortical outputs is associated with implicit learning (Pascual-Leone et al., 1994). The expansion of cortical representations, as measured by transcranial magnetic stimulation (TMS), is accompanied by a decrease in the cortical activation thresholds of muscles involved in the new motor task (Pascual-Leone et al., 1995). Moreover, mental rehearsal alone promotes the modulation of neural circuits (Pascual-Leone et al., 1995), indicating that generation of movements is not a fundamental requirement for motor cortical plasticity. These TMS ef- 
fects are modulated in a manner consistent with mechanisms of spike timing-dependent plasticity (STDP) (Wang et al., 1996). Changes in M1 during motor learning appear to involve longterm potentiation (LTP) of synapses (Rioult-Pedotti et al., 2000), mirroring plasticity mechanisms following injury.

Despite ample evidence of the capacity for plastic cortical reorganization, attempts to induce sustained motor output reorganization have been limited. We investigated whether and how the relationship between cortical efferent zones and forelimb muscles could be modulated by continuous activity-dependent feedback from forelimb muscles to motor efferent zones of other muscles. This was accomplished with a novel application of an autonomous head-fixed miniature computer called "neurochip" (Mavoori et al., 2005). Understanding the degree of flexibility in interactions between cortex and muscles is fundamental to understanding cortical control of movement. Targeted plasticity that exploits this flexibility could provide novel strategies for clinical treatments of motor impairment.

\section{Materials and Methods}

Subjects and behavioral task. Four male Macaca nemestrina were trained to perform a zero-torque wrist-holding task to provide consistent conditions for testing outputs evoked by intracortical microstimulation (ICMS). During training and testing sessions, animals sat quietly in a primate chair with the right forelimb restrained in a neutral resting position and the elbow bent at $90^{\circ}$. The hand was positioned around the central handle of a manipulandum that measured isometric torque about the wrist in four axes (flexion-extension, pronation-supination, radialulnar deviation, grip-release). A molded clam-shell attachment secured the hand within the device. A monitor in the booth displayed a tracking cursor for real-time feedback on the position and orientation of the manipulandum handle. Torques exerted upon the handle produced corresponding movements of the cursor.

The task in this experiment required that the subject exert no force on the manipulandum handle. A target box first appeared in the center of the monitor screen, corresponding to an isometrically neutral (zerotorque) position. The target box was only slightly larger than the tracking cursor, so any torques exerted upon the manipulandum handle resulted in a reset of the reward timer. The monkey was required to hold the tracking cursor within the target box for $3 \mathrm{~s}$ to trigger a reward of applesauce on a variable ratio schedule. Each trial was separated by a $3 \mathrm{~s}$ pause before the target box reappeared. Animals quickly learned to relax the forelimb muscles to keep the tracking cursor within the center target box to attain the maximum frequency of rewards. This design ensured that the motor system was in a consistent state of baseline inactivation across trials. This avoided any activation that would influence the cortically evoked output effects. Animals were monitored continuously with closed-circuit video. Trials were reset without reward if the animal failed to maintain the zero-torque position for the duration of the hold time.

Experimental paradigm. This experiment investigated the changes in motor output effects produced by activity-dependent conditioning (ADC) between a forelimb muscle and an unrelated motor efferent zone. The experiment consisted of three phases: preconditioning "baseline," conditioning, and postconditioning. Each phase involved testing sessions in the primate booth while the animal performed the holding task. Output effects evoked by trains of ICMS at motor cortex sites were documented during testing sessions. Output effects were quantified with ICMS train-triggered averages (TTA) of wrist torques and electromyography (EMG) of activated forelimb muscles (see below, Torque and EMG analysis).

Surgical procedures and implants. For the cortical implant surgeries, the animals received cephalexin $(25 \mathrm{mg} / \mathrm{kg})$, dexamethasone $(1 \mathrm{mg} / \mathrm{kg})$, and analgesics (ketoprofen $5 \mathrm{mg} / \mathrm{kg}$ plus buprenorphine $0.15 \mathrm{mg} / \mathrm{kg}$ ) and anesthetics (isoflurane $2 \%$, dexmedetomidine $0.05 \mathrm{mg} / \mathrm{kg}$ plus ketamine $5 \mathrm{mg} / \mathrm{kg}$ ). A craniotomy was performed at the location of the forelimb area of M1 (anterior $13 \mathrm{~mm}$, lateral $18 \mathrm{~mm}$ ). The location of forelimb M1 was confirmed with forelimb movements evoked by cortical surface stimulation. The dura was bonded to the pia with drops of cyanoacrylate to stabilize motion. A custom electrode array (Jackson and Fetz, 2007) was lowered into position above forelimb M1, such that the long axis of the microwires was oriented parallel to the bank of the precentral gyrus. Each microwire was manually advanced under direct visual guidance until it penetrated the pia. The solid tungsten wires were sufficiently rigid to avoid deformation during penetration of the pia. The array was enclosed and anchored to the skull and encased in a titanium chamber with methyl methacrylate. Animals were permitted to recover for 2 weeks before further procedures or experimentation. Animals received analgesics on a regular schedule during this interval.

During subsequent procedures, M1 output effects from the electrodes were documented under light ketamine sedation. Microwires were incrementally advanced down the bank of the precentral gyrus until evoked responses were identified. Each array consisted of individually moveable Teflon-coated tungsten microwires (50 or $127 \mu \mathrm{m}$; geometric surface area $1.96 \cdot 10^{-5} \mathrm{~cm}^{2}$ or $1.26 \cdot 10^{-4} \mathrm{~cm}^{2}$ ) cut at right angles to produce impedances ranging between 0.01 and $2 \mathrm{M} \Omega$ at $1 \mathrm{kHz}$ (Jackson and Fetz, 2007). The cortical surface area sampled by these arrays ranged between 16.4 and $24.3 \mathrm{~mm}^{2}$, centered on forelimb M1. Average interelectrode distances ranged between 0.57 and $1.14 \mathrm{~mm}$.

Short trains of ICMS delivered through each microwire activated contralateral forelimb muscles. Muscle activations were identified by visual inspection of fascicle contraction, manual palpation, joint movements, and EMG recordings via temporarily inserted needle electrodes. Muscles consistently activated by ICMS were implanted with pairs of transcutaneous EMG wires to record muscle activity chronically. EMG electrodes consisted of insulated, braided stainless steel wires (31 American wire gauge). Two wires spaced $1 \mathrm{~cm}$ apart were passed into the muscle bellies of targeted muscles after bending the tip to create a hook and backloading in $18 \mathrm{~g}$ hollow bore needles. Appropriate placement was confirmed by evoking muscle contractions with low-threshold electrical stimulation through the EMG wires $(0.5-2 \mathrm{~mA}, 30 \mathrm{~ms}$ train at $300 \mathrm{~Hz})$ and by monitoring EMG responses on an oscilloscope during ICMS testing. The wires were secured on the skin with drops of cyanoacrylate. Medical grade adhesive tape provided additional support. The forelimb was then wrapped in gauze padding for comfort. Finally, the forelimb and torso were protected with custom canvas jackets to prevent dislodgment of EMG wires. Animals received scheduled analgesics daily while EMG implants were in place. All procedures were approved by the Institutional Animal Care and Use Committee at the University of Washington.

ADC. Three motor output sites and their corresponding activated muscles were selected for each experiment on the basis of stable and discrete output effects evoked by short ICMS trains with the arm resting in a neutral position. Motor cortex sites and their activated muscles were designated as follows: one cortical site (Nrec) activated the forelimb muscle (Mrec) chosen for recording during conditioning; a second cortical site (Nstim), chosen for EMG-triggered stimulation during conditioning, activated a different muscle (Mstim); a third cortical site (Ctrl) and its activated muscle (Mctrl), were chosen as internal controls (Fig. 1a).

During the preconditioning baseline phase, the output effects in muscles and torques evoked from the three sites were documented. During the conditioning phase, activity of the Mrec muscle triggered stimulation of the Nstim cortical site via the continuous operation of a head-fixed computer, called neurochip (Mavoori et al., 2005; Jackson et al., 2006b). The neurochip was programmed as described below and activated at the beginning of the conditioning phase. The animal was then returned to the home cage. Throughout this phase the animal engaged in the usual repertoire of unrestrained volitional behaviors in the home cage, including reaching, feeding, and grooming. The home cage was populated with enrichment toys to encourage exploration and manipulation with the forelimb under investigation. Small treats were hidden within toys to encourage bimanual exploratory behaviors. For overnight sessions, the neurochip remained active during sleep. No abnormal forelimb movements or seizures were noted during conditioning in any of the experiments.

The neurochip contained the circuitry to record, digitize, and discriminate muscle activity and deliver recurrent intracortical stimulation in 
a ICMS testing before and after conditioning at each site individually
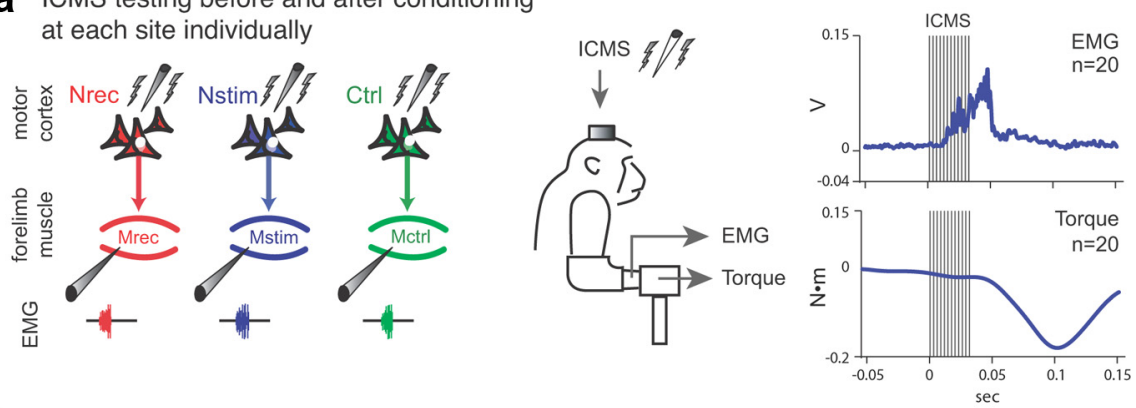

b Activity-dependent conditioning with $\mathrm{rBCl}$
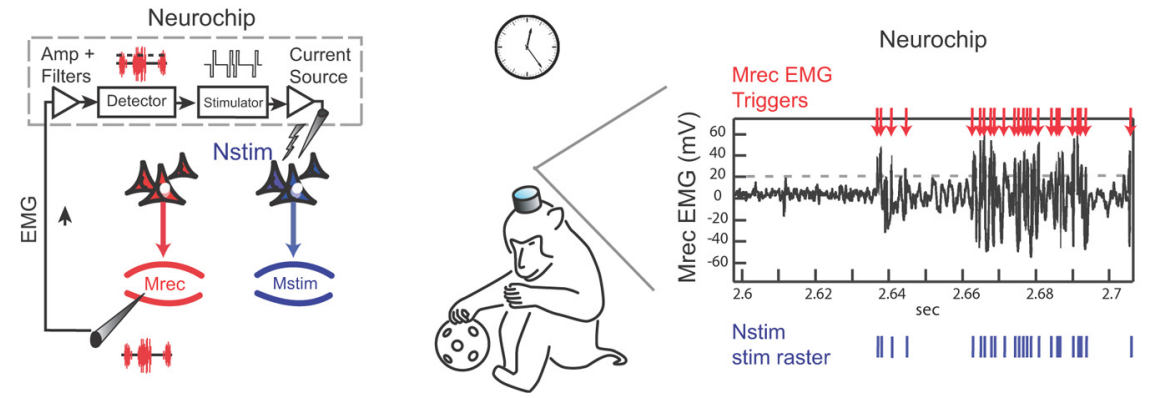

Figure 1. Conditioning paradigm. $\boldsymbol{a}$, ICMS testing before and after conditioning documented the muscle and torque responses at movement threshold for different cortical sites with the forelimb in a resting, neutral position. Right, Example TTAs of rectified EMG and torque responses evoked from one cortical site. $\boldsymbol{b}$, During ADC the neurochip discriminated Mrec raw EMG and delivered proportional intracortical subthreshold stimuli at cortical site Nstim while the animal engaged in unrestrained behavior. Right, Mrec EMG activity exceeding threshold (horizontal dashed line)-triggered current pulses noted in the Nstim raster (blue).

real-time (Mavoori et al., 2005; Jackson et al., 2006b). The neurochip recorded analog EMG signals that were bandpass filtered $(500-5 \mathrm{kHz})$ with a four-pole multiple feedback Butterworth filter, and then amplified $\times 1500$. After analog-to-digital conversion (at $11.7 \mathrm{ksps}$ ) the signal passed to the first of two "Programmable Systems-on-a-Chip" (Cypress Microsystems). An 8-bit microprocessor core ran a signal discrimination algorithm on the multiunit EMG activity. The algorithm first identified threshold crossing and then discriminated signals that passed through two adjustable time-voltage windows. Acceptance pulses were used as "triggers" that activated a control routine that ran a stimulator board at a predetermined stimulation ratio (defined as acceptance pulses: stimulus pulses). The neurochip was programmed to deliver subthreshold conditioning stimulation (43-56\% of resting movement threshold) that did not disrupt normal movements. Animals were returned to the home cage environment that allowed unrestricted movements.

The neurochip was tested in separate sessions for its ability to autonomously record and discriminate multiunit muscle activity, as well as generate stimulation triggers for up to $30 \mathrm{~h}$. The neurochip was programmed to record $21 \mathrm{~ms}$ of raw data every $8.8 \mathrm{~min}$ to verify the quality of EMG signal and discrimination. Stimulation rates were recorded for successive $100 \mathrm{~ms}$ bins. Two additional channels of differential EMG (2 ksps) were also recorded. EMG data were bandpass filtered between $20-2000 \mathrm{~Hz}$, with $250 \times$ amplification followed by full-wave rectification. Adjustable parameters to optimize signal detection and stimulation delivery included: DC offset, signal gain, detection threshold, discrimination windows (amplitude and delay), stimulation ratio, and stimulation intensity. Stimulation was disabled for a $4 \mathrm{~ms}$ refractory period after each stimulus pulse to prevent artifact-triggered stimulation during conditioning.

The conditioning phase concluded with a testing session in the primate booth to document the output effects of Nrec, Nstim, and Ctrl. The neurochip was deactivated immediately before this testing session. Subsequent postconditioning testing sessions documented the extinction of any conditioned changes in output effects.

Experiments were designed to test the capacity for reorganization between targeted pairs of M1 sites and the muscles that they activated. Three of the five experiments tested the interactions between forelimb "functional agonists" (Experiments 3-5; Table 1). Functional agonists were defined as muscles that acted cooperatively during ethologically relevant behaviors, such as supinating the forelimb and extending the wrist, as might occur when plucking a banana from a tree. Functional agonists exerted distinct actions at a given joint when activated individually, and therefore were not considered anatomical agonists. Two experiments tested interactions between anatomical antagonists, namely muscles that exerted opposing actions at a given joint, as in the case of extensors and flexors.

Torque and EMG analysis. Output effects of the cortical efferent zones were quantified with TTAs of wrist torques and forelimb EMG beginning 2 weeks after electrode implantation. ICMS thresholds for each site were determined during each experimental session. Thresholds were defined as the lowest current intensity that evoked wrist movement at least $50 \%$ of the time while the animal performed the holding task. Selecting this current threshold meant that up to half of the ICMS trains did not result in overt movements. Stimuli consisted of a 39 ms train of symmetric, negative-positive biphasic square wave pulses $\left(0.2 \mathrm{~ms}\right.$ phase $\left.{ }^{-1}\right)$ at $333 \mathrm{~Hz}$. Thresholds were $59.09 \pm 23.89 \mu \mathrm{A}$ for $50 \mu \mathrm{m}$ wires (animals $\mathrm{V}, \mathrm{Y}$, and $\mathrm{Z}$ ) and $96.67 \pm$ $29.15 \mu \mathrm{A}$ for $127 \mu \mathrm{m}$ wires (animal A). The estimated radius of tissue activated by direct current spread was 243 and $311 \mu \mathrm{m}$, respectively, based on the equation $r^{2}=i / k$, where $r$ is the radius of activated tissue (in millimeters), $i$ is current (in microamperes), and $k$ is a constant, assumed to be 1000 (Stoney et al., 1968). Polysynaptic effects recruited by temporal summation during stimulus trains are not limited to this radius (Jankowska et al., 1975).

Stimulus TTA of torque responses were compiled by averaging trials over a peristimulus window $( \pm 500 \mathrm{~ms})$. Full-scale torque responses were calibrated at $0.5 \mathrm{~N} \cdot \mathrm{m} / 5 \mathrm{~V}$, and digitized at $5 \mathrm{kHz}$. TTAs at movement threshold were transformed into 3D "torque space" (Fig. 2). Torque TTAs were aligned on the center point origin in $3 \mathrm{D}$ plots, corresponding to the zero-torque position on all axes. This permitted serial comparisons across sites and experimental sessions. Curved lines represent mean torque trajectories evoked by ICMS at a site during an experimental session. Straight arrows represent mean torque vectors over the first 120 $\mathrm{ms}$ of the torque trajectories.

TTAs were also generated at subthreshold and suprathreshold current intensities (Fig. 3). Torque vectors and trajectories over a range of stimulus intensities at a single cortical site are illustrated. Torque vectors were projected onto the major planes of torque deflections (Fig. 3, arrow shadows) to permit angular measurements between torques evoked from separate cortical sites. Subsequent comparisons were made on the major planes.

Peristimulus TTAs of rectified EMG activity were compiled by averaging trials during the same interval as torque TTAs ( $\pm 500 \mathrm{~ms})$. Some raw EMG recordings showed ICMS artifacts, consisting of stereotypic waveforms corresponding to the stimulus delivery times. Artifact correction was performed using linear interpolation across $0.8 \mathrm{~ms}$ peristimulus window. Artifact correction was aided by the fact that ICMS stimulus frequency, pulse width, time gate, and interstimulus interval were held constant throughout the testing periods.

EMG TTAs were used to calculate the mean normalized response, integrated area under the response curve, maximum response value, and response duration (Fig. 4). To identify responses, means and confidence intervals ( \pm 3 SDs) of full-scale rectified EMG were calculated from the $500 \mathrm{~ms}$ interval preceding stimulus onset. The "onsets" of evoked EMG responses were determined with a sliding $6 \mathrm{~ms}$ detection window. Re- 
Table 1. Conditioning stimulation parameters

\begin{tabular}{|c|c|c|c|c|c|c|c|c|c|}
\hline \multirow[b]{2}{*}{ Exp } & \multirow[b]{2}{*}{ Figure } & \multirow[b]{2}{*}{ Cx session } & \multicolumn{3}{|c|}{ Conditioning parameters } & \multirow[b]{2}{*}{$\mathrm{CE}\left({ }^{\circ}\right)$} & \multicolumn{3}{|c|}{ Direction of mean torque } \\
\hline & & & Current $(\mu \mathrm{A})$ & Frequency $(\mathrm{Hz})$ & Duration (h) & & $\operatorname{Nrec}\left({ }^{\circ}\right)$ & Nstim $\left({ }^{\circ}\right)$ & Control $\left({ }^{\circ}\right)$ \\
\hline \multirow[t]{2}{*}{1} & $7 b$ & 1 & 39 & 5.3 & 23.5 & 91.8 & -13.3 & -74.3 & 67.1 \\
\hline & & 2 & 39 & 5.2 & 24.5 & 47.7 & 30.8 & -71.6 & 51.8 \\
\hline \multirow[t]{3}{*}{2} & $7 e$ & 1 & 39 & 7.3 & 5.9 & 78.2 & 232.4 & 288.9 & 53.4 \\
\hline & & 2 & 39 & 2.7 & 9.1 & 52 & 206.2 & 278.1 & 89.6 \\
\hline & & 3 & 39 & 4.3 & 12.1 & 74.6 & 228.8 & 253.1 & 54.5 \\
\hline \multirow[t]{4}{*}{3} & 8 & 1 & 44 & 9 & 0.4 & 46.7 & 231.2 & 204.3 & 176.5 \\
\hline & & 2 & 44 & 9.1 & 22.8 & 58.5 & 219.4 & 215.4 & 165.5 \\
\hline & & 3 & 44 & 6.7 & 24 & 96 & 181.9 & 192.5 & 176.8 \\
\hline & & $4^{\S}$ & 44 & 9 & 0.4 & 17.7 & 260.9 & 205.7 & 170 \\
\hline \multirow[t]{6}{*}{4} & 9 & 1 & 39 & 4.2 & 3.1 & 59.1 & -9 & 99.7 & 131.2 \\
\hline & & 2 & 39 & 1.9 & 21 & 82.5 & 14.4 & 66.9 & 131.7 \\
\hline & & 7 & 18 & 2.1 & 23.5 & 72 & 3.9 & 83.1 & 143.2 \\
\hline & & 8 & 18 & 1.4 & 22.6 & 70 & 1.9 & 103.4 & - \\
\hline & & 9 & 18 & 1 & 25.4 & 68.8 & 1.7 & 86.2 & - \\
\hline & & 10 & 18 & 0.5 & 23.3 & 68.6 & 1.5 & 82.7 & - \\
\hline \multirow[t]{3}{*}{5} & - & $1^{\S}$ & 39 & 5 & 0.3 & 1 & 178.1 & 124 & 162 \\
\hline & & 2 & 39 & 8.4 & 0.3 & 175.1 & 4 & 138 & 164 \\
\hline & & $3^{\S}$ & 39 & 8 & 0.3 & 7.1 & 172 & 114 & 163 \\
\hline
\end{tabular}

Conditioning parameters for five experiments are shown. Conditioning sessions are numbered sequentially. ${ }^{\S}$, Pseudoconditioning sessions; ${ }^{*}$, loss of microwire due to mechanical failure. $\mathrm{Cx}$, Conditioning; $\mathrm{CE}$, conditioning effect.

sponses were defined by values exceeding 3 SDs above the baseline mean. The onset of such responses was defined as the beginning of the sliding window. Similarly, EMG response "offset" was defined as the time that the TTA fell within the confidence interval of baseline for 6 ms (Fig. 3). Correspondence between TTA of EMG and torque data is illustrated in Figure 5. Significant responses were compared before and during conditioning. One-way ANOVA was performed on the mean normalized EMG responses for each muscle during the response envelope, grouped by experiment phase. Data were normalized by stimulus intensity to control for changes in ICMS movement thresholds between sites and across sessions.

Experimental controls. Conditioning sessions included control sites to determine whether the conditioning effect was cortically site specific (i.e., occurring only at Nrec) and input specific (i.e., occurring only during $\mathrm{ADC}$ ). Within-session controls consisted of a neighboring Ctrl site that was located in close proximity to Nrec and Nstim (Fig. 6). ICMS at the Ctrl site was performed during each testing session to detect any nonspecific changes in M1 output effects attributable to stimulation alone, or to global changes in excitability or motivational states. The distances between the cortical entry sites were measured to determine whether a spatial bias was present that would influence current spread during conditioning or ICMS testing. There were no significant differences in the separation between pairs of cortical sites (Nstim and Ctrl, $1.9 \pm 1.79 \mathrm{~mm}$; Nstim and Nrec, $2.68 \pm 0.92 \mathrm{~mm}$; and Nrec and Ctrl, $3.11 \pm 1.44 \mathrm{~mm}$; ANOVA, $F_{(2,14)}=0.914, p=$ n.s. $)$.

Additional controls were performed during "pseudoconditioning" sessions to determine whether the conditioning effects were input specific. Pseudoconditioning consisted of stimulation delivered to Nstim sites that demonstrated the capacity for changes in motor output effects during ADC from Mrec. Pseudoconditioning sessions were interleaved in block design with ADC, either following or preceding conditioning sessions. Stimulation parameters for pseudoconditioning were matched to ADC across the following parameters: location (Nstim), stimulation intensity, polarity, pulse width, mean frequency, total number of stimuli, duration, and behavioral state. Due to the limited programming capacity of the neurochip computer, pseudoconditioning was delivered at constant frequency, without the phasic modulation observed during EMGtriggered conditioning. Therefore pseudoconditioning had no consistent temporal correlation with forelimb muscle activity. Similar methods of delivering matched stimulation, unassociated with forelimb movement, have been used to validate the requirement of activity dependence on motor cortex reorganization (Porter et al., 2012).

Statistics. In addition to the statistics mentioned above, we computed summary statistics. Statistical analyses between three or more independent groups with normal distributions were performed with two-tailed ANOVA. Levene's test for homogeneity of variance was performed to ensure equal variance between sample groups. Two-tailed independent samples T tests $(t)$ were performed for comparisons between two groups with normal distributions. To correct for cumulative Type I statistic errors, Bonferroni's post hoc test was performed for all ANOVA procedures. Groups that reached a familywise $\alpha$ value of 0.05 in post hoc tests are noted. ANOVA statistics are reported with the $F_{x, y}=$ value, where $x$ 


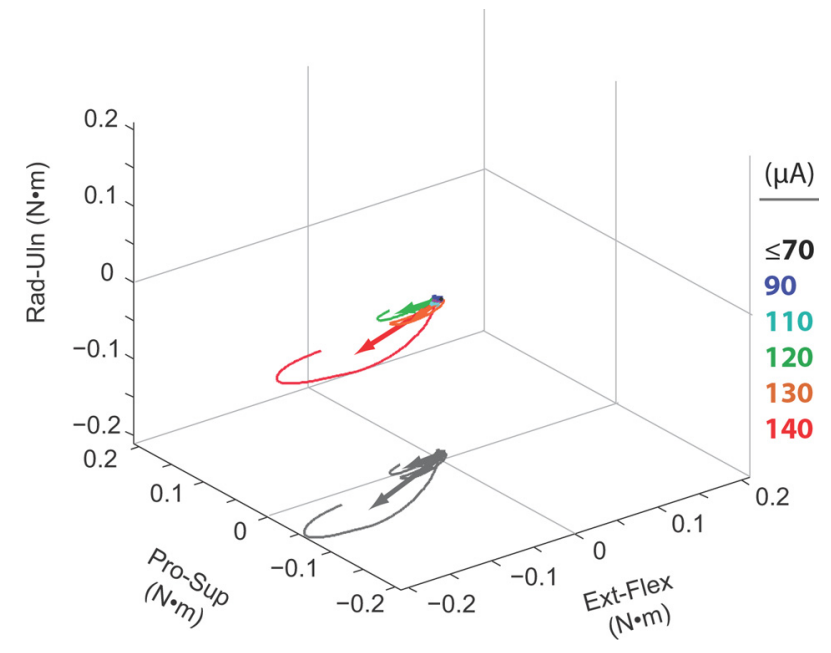

Figure 3. ICMS torque responses as a function of stimulus intensity. Torque trajectories (colored curved lines) and mean torque vectors (colored straight lines) for different stimulus intensities delivered to a cortical site associated with forelimb extension plotted in $3 \mathrm{D}$ torque space. Data represent $120 \mathrm{~ms}$ following stimulus onset. To permit serial comparisons and angular measurements between torques, shadows of the torque vectors are projected (in gray) onto the major plane capturing the torque directions. Subsequent comparisons are made on the major planes.
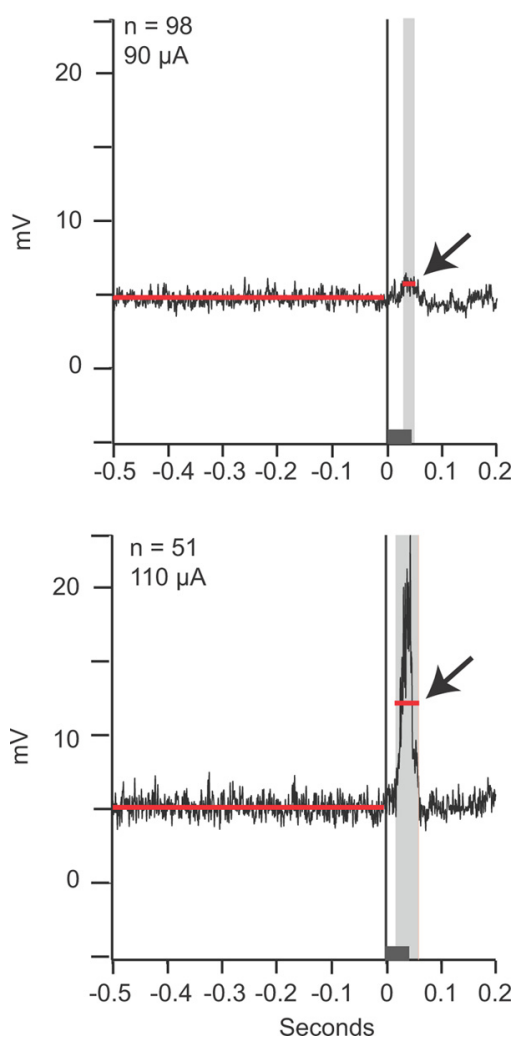

Figure 4. EMG Response to ICMS. TTAs of rectified EMG evoked during ICMS at two stimulation intensities in animal $A$. Response onsets were defined as times when the mean response over a $6 \mathrm{~ms}$ sliding window exceeded 3 SDs above baseline mean. Baseline means were calculated from the $500 \mathrm{~ms}$ immediately preceding stimulus onset (vertical line). Stimulus train is shown as horizontal dark gray bar. Higher stimulation intensity evokes larger TTA response (arrows). Red bars show baseline and response mean.

represents the degrees of freedom between groups and $y$ represents the degrees of freedom within groups. For each experiment, TTA data for each cortical site were grouped by experiment phase (baseline, conditioning, and postconditioning), and factored on the direction of mean torque per session for each cortical site. For summary statistics, data across all experiments were combined grouping by phase. Pearson's correlation coefficients $(r)$ were performed to test the strength of linear associations between continuous variables. Nonparametric tests were performed with $\chi^{2}$ statistic $\left(\chi^{2}\right)$ for categorical variables. Significance for all statistics was set at the 0.05 level. Error bars for all figures indicate $95 \%$ confidence intervals, unless otherwise stated. Additional statistical notation is included, where appropriate, within the figure legends.

\section{Results}

Results were obtained from four M. nemestrina. The data presented here summarize 1025 ICMS recordings from 108 sessions collected over a 24 month period. Of 108 sessions, there were 22 conditioning sessions, comprising 5 conditioning experiments and 2 pseudoconditioning experiments. ADC generated reorganization of motor output effects in all five conditioning experiments.

\section{Artificial connection between antagonist muscles}

Representative results for two conditioning experiments pairing anatomical antagonist muscles in separate animals are shown in Figure 7 . In the first, conditioning-paired two-digit antagonists: activity of a flexor muscle (flexor digitorum superficialis = Mrec) triggered recurrent intracortical stimulation at a cortical site (Nstim) associated with an extensor muscle (extensor digitorum communis; $\mathrm{EDC}=$ Mstim) (Fig. 7a). Thus, conditioning created the following recurrent feedback loop: Nrec cortex $\rightarrow$ Mrec muscle $\rightarrow$ Neurochip $\rightarrow$ Nstim cortex.

The mean baseline torque trajectories (curved lines) and average torque vectors (straight line segments) for each cortical site are shown in the plane that captured the dominant torque directions (Fig. 7a, Baseline). Following $24 \mathrm{~h}$ of ADC, M1 output effects at these sites were documented (Fig. $7 a$, Conditioning). The Nrec torque vector (red) shifted $92^{\circ}$ toward the Nstim vector (blue) following a single ADC session. Nstim and Ctrl vectors remained stable, indicating that the conditioning effect observed at Nrec was site specific and not a generalized phenomenon due to arousal, sensitization, or stimulation alone. The angular rotation of Nrec torque vectors, termed the "conditioning effect," was associated with a substantial shift in the initial segment of the torque trajectory (Fig. $7 a$, gray arrows), which was now aligned with the Nstim torque. The stimulus intensity during ADC was $39 \mu \mathrm{A}$ ( $43 \%$ of resting movement threshold) and did not evoke any observed movements during conditioning. The stimulation rate triggered by muscle activity during this session fluctuated (Fig. $7 c$ ), with a mean rate of $5.2 \pm 4.5 \mathrm{~Hz}$.

The direction of mean torque vectors across serial sessions is plotted in Figure $7 b$. ADC sessions are highlighted with shaded intervals. During each ADC session, Nrec demonstrated a robust conditioning effect (CE). The rotation of Nrec torque vector illustrated in Figure $7 a$ is indicated on the plot of the direction of mean torque with *. The mean CE across three $24 \mathrm{~h}$ conditioning sessions was $61.0 \pm 26.7^{\circ}$ (Fig. $7 b$; Baseline vs ADC, $t=4.618, p<$ $0.01)$. Mean output effects from the neighboring Ctrl site, separated from Nrec by $1 \mathrm{~mm}$ (Fig. $7 h$ ), and the Nstim site deviated negligibly from baseline after conditioning $(t=-1.024, p=$ n.s.; $t=1.454, p=$ n.s.; resp.). The observed stimulation rate triggered by muscle activity across all three ADC sessions fluctuated significantly, with bursts of stimulation reflecting the profile of the muscle activity during free volitional movements (Fig. $7 c$ ); the mean stimulation rate for all three ADC sessions was $6.7 \pm 6.8 \mathrm{~Hz}$. 


\section{Artificial connection between}

functional agonist muscles

In a second animal we created an artificial connection between functional agonists, defined as pairs of muscles that contracted cooperatively during ethologically relevant forelimb movements, but did not produce purely synergistic or antagonistic actions across a joint when contracting individually. EDC (Mrec) activity triggered stimulation of a cortical site (Nstim) that activated the forelimb supinator (Mstim). Conditioning over five sessions resulted in a robust and rapid shift in the evoked torques of the Nrec cortical site (Fig. 7e$g)$. The mean $\mathrm{CE}$ was $73.3 \pm 16.6^{\circ}$ in the direction of Nstim (ANOVA, $F_{(2,8)}=$ $33.094, p<0.001$, post hoc tests of conditioning vs baseline, $p<0.001$ ). Mean torque directions evoked from the Ctrl and Nstim sites did not change significantly across the conditioning block (ANOVA, $F_{(2,8)}=0.245, p=$ n.s.; ANOVA, $F_{(2,9)}=1.694, p=$ n.s.; resp.), although there was more variability in Nstim responses. The absence of a significant net change in the mean torque at the Ctrl site eliminated the possibility of modulation related to direct stimulation alone. The mean stimulation rate during ADC was $6.9 \pm 8.9 \mathrm{~Hz}$ and the stimulus intensity $(90 \mu \mathrm{A})$ was $43 \%$ of movement threshold. The stimulation pattern was highly variable, reflecting variations in Mrec EMG during unrestrained volitional forelimb movements. The conditioned changes in Nrec torque output returned to baseline within $9.8 \mathrm{~h}$ after the end of conditioning.

\section{Requirement of activity-dependent stimulation}

To test the hypothesis that activitydependent stimulation was necessary and sufficient to induce reorganization in these experiments, we performed additional control experiments in which stimulation of Nstim unrelated to muscle activity, termed pseudoconditioning, was alternated with ADC. Pseudoconditioning was identical to ADC with respect to the cortical stimulation site, current intensity, polarity, pulsewidth, mean frequency, duration, absolute stimulus counts, and behavioral condition - with one exception - unrelated stimulation was delivered at a constant frequency, with no correspondence to the activity of the forelimb muscles.

An example of these control experiments is illustrated in Figure 8. Successful conditioning was first established by associating supinator muscle activity with stimulation of an M1 site that activated the digit extensor, EDC. A significant conditioning effect of $46.7^{\circ}$ was observed within $25 \mathrm{~min}$ of the onset of ADC (Fig. $\left.8 a,{ }^{*}\right)$. By the second and third ADC sessions, the direction of Nrec and Nstim vectors did not differ statistically. The mean CE was $67.1^{\circ}$ (Baseline vs ADC, ANOVA, $F_{(2,6)}=14.119, p<0.005$ ). The CE was then extinguished in the presence of normal spontaneous activity without artificial feedback during unrestrained

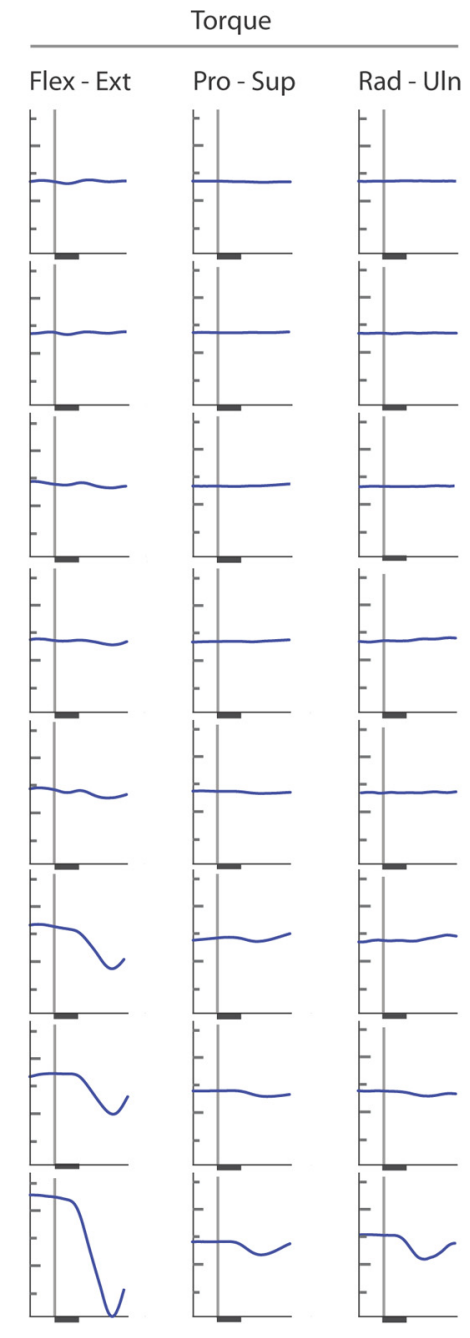

\section{EMG}

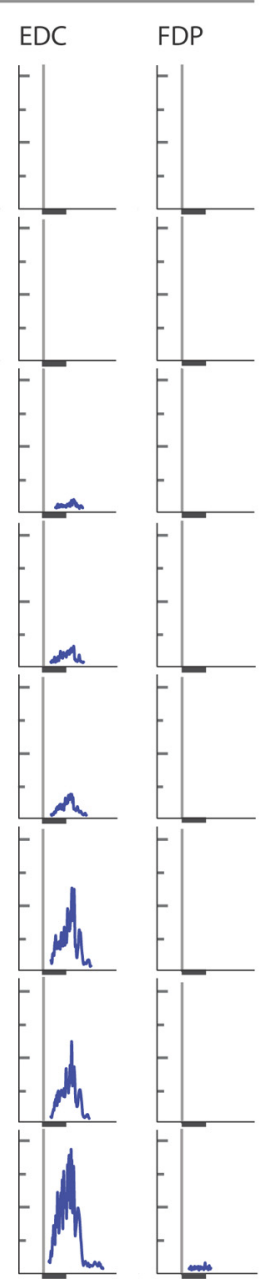

Figure 5. ICMS responses from a cortical site associated with forelimb extension. Muscle responses and torque responses are shown for different stimulus intensities delivered at a single cortical site in animal A. Torque responses, shown at the right, increase stimulation intensities but failed to generate consistent torques. Horizontal gray bars represent stimulus trains and train onset shown as vertical line. Calibration lines represent $50 \mathrm{mV}$ (EMG) or $0.05 \mathrm{~N} \cdot \mathrm{m}$ (torque). ED 4/5, extensor digitorum digits 4 and 5 ; EDC, extensor digitorum communis; FDP, flexor digitorum profundus; Rad, radial deviation; Uln, ulnar deviation; Pro, pronation; Sup, supination; Flex, flexion; Ext, extension.

movements. During pseudoconditioning PC, using stimulation rates identical as in ${ }^{*}$, no reorganization was observed (Fig. $8 a$, arrow; Baseline vs PC, $t=1.437, p=$ n.s.). Additional experiments interleaved ADC between blocks of pseudoconditioning, with similar results (Table 1).

\section{Effective conditioning parameters}

We tested the limits and sustainability of plasticity by switching artificial feedback "on" and "off" over repeated conditioning blocks in a separate animal (Fig. 9). A robust conditioning effect of $59^{\circ}$ was documented following a $3.2 \mathrm{~h}$ initial conditioning session (Fig. $9 b, *)$. This shift was accompanied by a significant change in the direction of the initial segment of Nrec torque trajectories (Fig. $9 a$, arrow) and a reversal of the direction of the trajectory trace at its inflection point (Fig. $9 a, \dagger$, Conditioning). To examine the effect of recruiting fewer cortical neurons during conditioning, the stimulation intensity during the conditioning block, ADC-1, was decreased from 39 to $18 \mu \mathrm{A}$ (56 and 26\% movement threshold, resp.) following the second session. No change in $\mathrm{CE}$ during the subsequent three sessions was noted, 
a

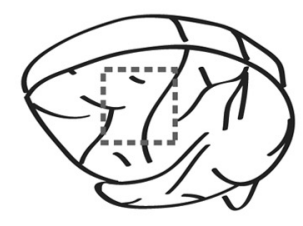

C

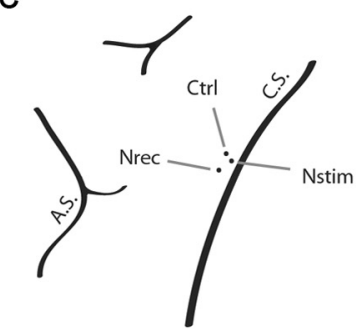

e

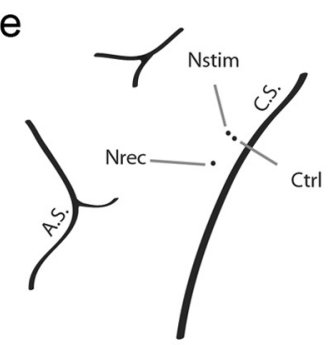

b

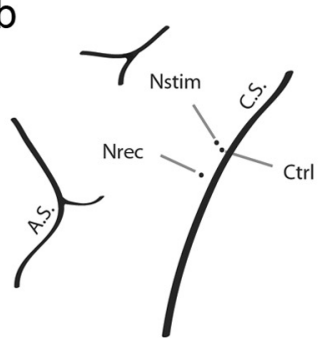

d

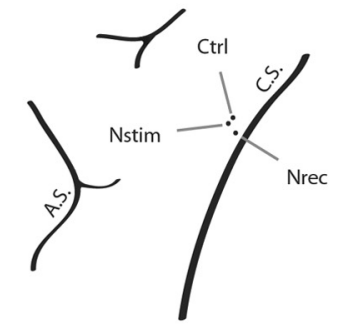

f

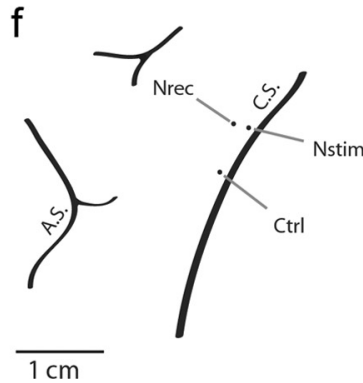

Figure 6. Location of microwire tracks during conditioning experiments. $\boldsymbol{a}$, Schematic of lateral cerebral hemisphere. $\boldsymbol{b}$, Experiment 1, animal Z.c, Experiment 2, animal Y.d, Experiment 3, animal Z. e, $\boldsymbol{f}$, Experiments 4 and 5 , animal A. Experiment numbers correspond to table entries.

effectively ruling out extensive local current spread as an explanation of reorganization of output effects. The mean CE during the first conditioning block was $>70^{\circ}$ (difference of mean torque directions during conditioning, $-68.1 \pm 6.9^{\circ}$; direction of mean torque before conditioning, $6.3 \pm 11.7^{\circ} ; t=-11.1, p<0.001$ ). The CE was reduced with spontaneous activity in the absence of artificial feedback within $20 \mathrm{~h}$.

A second block, ADC-2, tested the sustainability of the CE by introducing intermittent conditioning with a decreasing intermittent reinforcement schedule after the CE had been fully established. To achieve this, the ratio of intracortical stimuli-to-EMG triggers during conditioning was decreased incrementally from 1:4 to 1:16, while holding current intensity constant. The mean $\mathrm{CE}$ in block 2 remained highly significant $\left(\mathrm{ANOVA}, F_{(2,11)}=\right.$ $7308.8, p<0.001$; Fig. 9), and was similar to the mean of the first block $\left(\mathrm{CE}_{\text {block } 1}, 74.4^{\circ}\right.$; $\mathrm{CE}_{\text {block } 2}, 68.2^{\circ}$, Bonferroni post hoc test, $p=$ n.s.). Interestingly, the CE following intermittent reinforcement persisted for one session $21 \mathrm{~h}$ after the end of ADC before returning to baseline levels.

\section{Nstim movement thresholds}

To examine whether repeated subthreshold stimulation during conditioning affected movement thresholds during subsequent ICMS testing, Nstim thresholds were compared across experiment phases. In a previous experiment, movement thresholds were followed for $60 \mathrm{~d}$ in a separate animal with a microwire array identical to those used in the present study (our unpublished

observations). Thresholds were observed to fluctuate independently over time. To control for this variability, Nstim thresholds were normalized by baseline thresholds for each experiment. There were no differences in the Nstim mean normalized thresholds among baseline, conditioning, postconditioning, and pseudoconditioning phases (ANOVA, $F_{(3,45)}=1.141, p=$ n.s.).

\section{Relationship between conditioning effect and stimulation parameters}

To determine which, if any, of the conditioning parameters predicted the magnitude of the $\mathrm{CE}$, correlation analyses were run on the variables of stimulation frequency $(\mathrm{Hz})$, stimulation intensity $(\mu \mathrm{A})$, conditioning duration $(\mathrm{h})$, and total number of stimulation pulses delivered $(n)$. The CE was not correlated with rate of stimulation per session $(r=0.147, p=$ n.s. $)$. There was no linear association between the mean stimulation rate and the magnitude of the CE. The mean $\mathrm{CE}$ for $\mathrm{ADC}$ was $74.2 \pm 26.1^{\circ}$, with a range of 43.6 to $175.1^{\circ}$. This mean effect was relatively consistent over a range of average stimulation frequencies, from 0.52 to 10.29 Hz. The mean CE was compared with total number of stimulations $(n)$, the product of the mean stimulation rate, and duration of conditioning. No significant correlations were observed between CE and $n(r=-0.175, p=$ n.s. $)$. Similarly, the CE was independent of the total number of stimuli delivered across all ADC sessions $(r=0.337, p=0.07)$, beyond the minimum value of 5940 stimuli delivered in one 25 min session.

The CE was not correlated with the duration of individual conditioning sessions $(r=-0.292, p=$ n.s.), or the cumulative duration of conditioning across all preceding sessions. The conditioning durations per session ranged broadly, from $25 \mathrm{~min}$ to $>24 \mathrm{~h}$. Conditioning for only $25 \mathrm{~min}$ resulted in significant CE, consistent with the rapid onset of reorganization noted in other plasticity studies (see Discussion). These effects were as robust as the CE observed after $24 \mathrm{~h}$ of conditioning. Thus, in this case, conditioning beyond $25 \mathrm{~min}$ conferred no additional CE. There were no identifiable trends relating $\mathrm{CE}$ and conditioning duration.

The CE was analyzed as a function of stimulation intensity during conditioning sessions. There were no significant differences in the magnitude of the $\mathrm{CE}$ across different stimulation intensities (ANOVA, $F_{(4,17)}=0.97, p=$ n.s.), which ranged from 18 to $59 \mu \mathrm{A}$. This suggests that the shift in torque vectors measured during $\mathrm{CE}$ was not related to differences in the spread of current at different stimulation intensities. The mean CE for the highest stimulation intensities (59 $\left.\mu \mathrm{A}, 78.7 \pm 16.4^{\circ}\right)$ was nearly identical to the lowest intensities $\left(18 \mu \mathrm{A}, 74.2 \pm 9.3^{\circ}\right)$ necessary to maintain the $\mathrm{CE}$. The rate of extinction of the $\mathrm{CE}$ was not correlated with the conditioning parameters of conditioning duration, stimulation intensity, or mean stimulation frequency during conditioning.

In addition to quantifying torque responses, we documented the muscle responses evoked with ICMS trains at each cortical site before and immediately following conditioning sessions in a subset of muscles. Significant changes in TTAs of normalized EMG responses were found across experimental phases and between efferent zones. The direction of EMG changes was very variable. Muscle changes were categorized into the nominal variables of increased, decreased, or unchanged. Compiling muscle responses by cortical site across all experiments revealed that Mrec responses evoked from ICMS at Nrec increased following conditioning $\left(\chi^{2}=6.4, p<\right.$ 0.05). Responses from Nstim and Ctrl were more variable, with equal proportions of muscle responses increasing, de- 
a
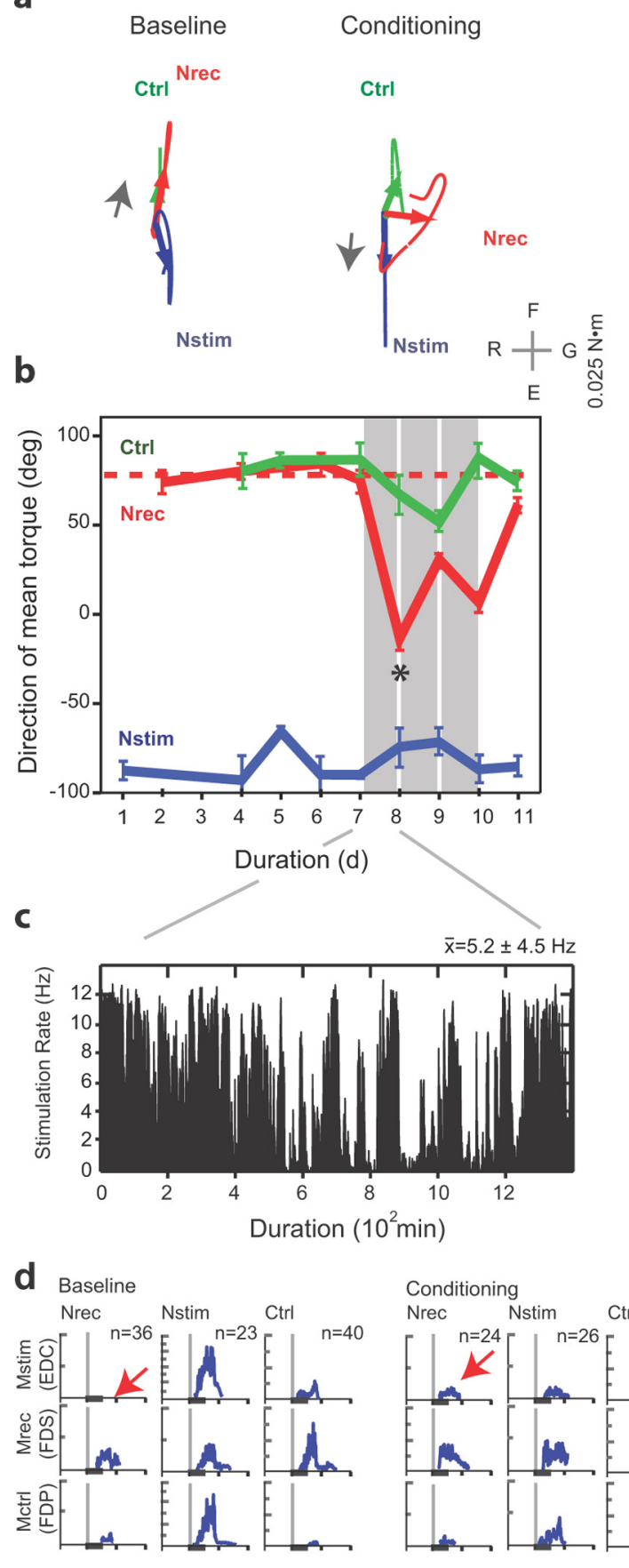

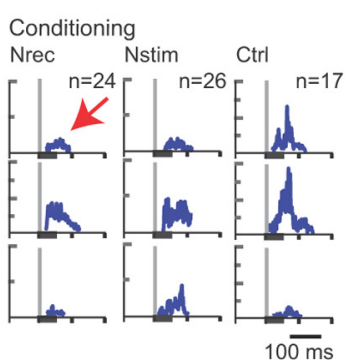

e

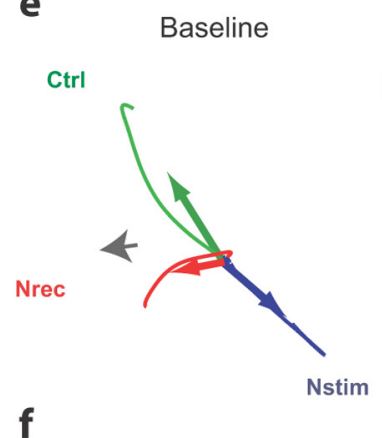

Conditioning

Ctrl

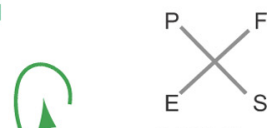

$0.025 \mathrm{~N} \cdot \mathrm{m}$

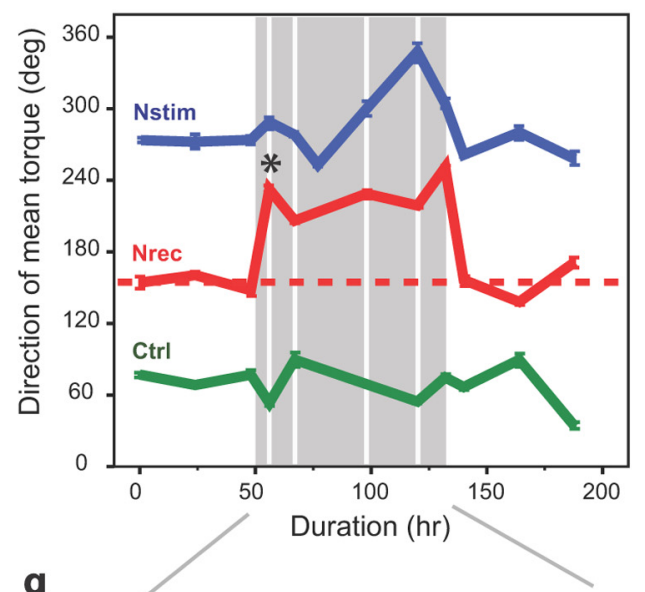

$\mathbf{g}$

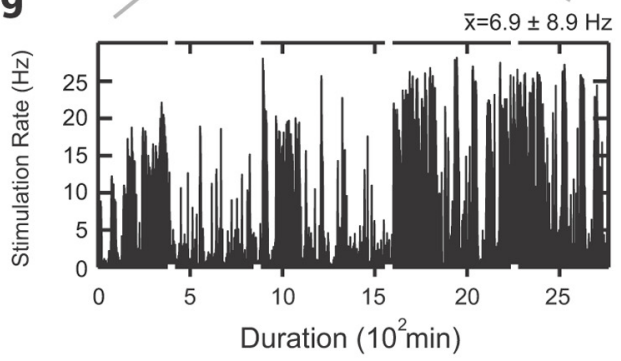

h

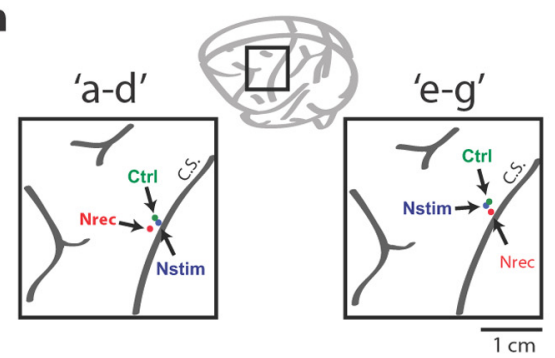

Figure 7. Motor cortex output reorganization during conditioning. $\boldsymbol{a}$, Average wrist torque vectors (colored arrows) and trajectory traces (curved lines) plotted in torque space in the plane that captured dominant torque directions during ICMS at three cortical sites. Nrec vector (red arrow) shifts $92^{\circ}$ after the first $24 \mathrm{~h}$ conditioning session (* in $\boldsymbol{b}$ ). The initial $50 \mathrm{~ms} \mathrm{segment}$ of the Nrec trajectory (indicated by gray arrows) reverses direction following Conditioning, aligning with the trajectory of Nstim. F, flexion; E, extension; $G$, grip; $R$, release. $\boldsymbol{b}$, Direction of mean torques over 11 consecutive days. The mean conditioning effect during $3 \mathrm{~d}$ of conditioning (gray regions) was $61.0 \pm 26.7^{\circ}$. Dashed red line shows mean Nrec at baseline. Error bars indicate SEM. $c$, Rates of stimuli triggered by Mrec muscle activity during free movement during first conditioning session. Mean rate for all three conditioning sessions was $6.9 \pm 6.8 \mathrm{~Hz}$. Data concatenated in $100 \mathrm{~ms}$ bins. $\boldsymbol{d}$, Muscle responses evoked by ICMS. Red arrows indicate the appearance of responses in EDC (Mstim) evoked by ICMS at Nrec following a single conditioning session (* in $\boldsymbol{b})$. $\boldsymbol{e}-\boldsymbol{g}$, A separate conditioning experiment in another animal. Following $5.8 \mathrm{~h}$ of conditioning, Nrec torque vectors shifted $78.2^{\circ}$ toward Nstim responses ${ }^{*}$ in $\left.\boldsymbol{f}\right)$. P, pronation; $S$, supination; $F$, flexion; $E$, extension. $\boldsymbol{f}$, Mean conditioning effect was $73.3 \pm 16.6^{\circ}$ across five conditioning sessions. $\boldsymbol{g}$, Stimulation rates (over 1 min bins) during all ADC sessions. $\boldsymbol{h}$, The relative location of microwires for the two animals. C.S., central sulcus.

creasing, or remaining unchanged $\left(\chi^{2}=1.6, p=\right.$ n.s.; $\chi^{2}=$ $1.5, p=$ n.s., resp.). Figure $7 d$ illustrates changes in muscle responses: after delivering conditioning stimuli to Nstim triggered from Mrec (FDS), ICMS stimulation of Nrec evoked significantly larger responses of the Mstim muscle (EDC)
(ANOVA, $F=6.671, p=0.002)$. Conditioning was also associated with significant decreases in Mstim responses evoked by ICMS at Nstim (ANOVA, $F=79.738, p<0.001$ ) and no change in responses evoked from the neighboring Ctrl (ANOVA, $F=2.079, p=$ n.s.). 
a

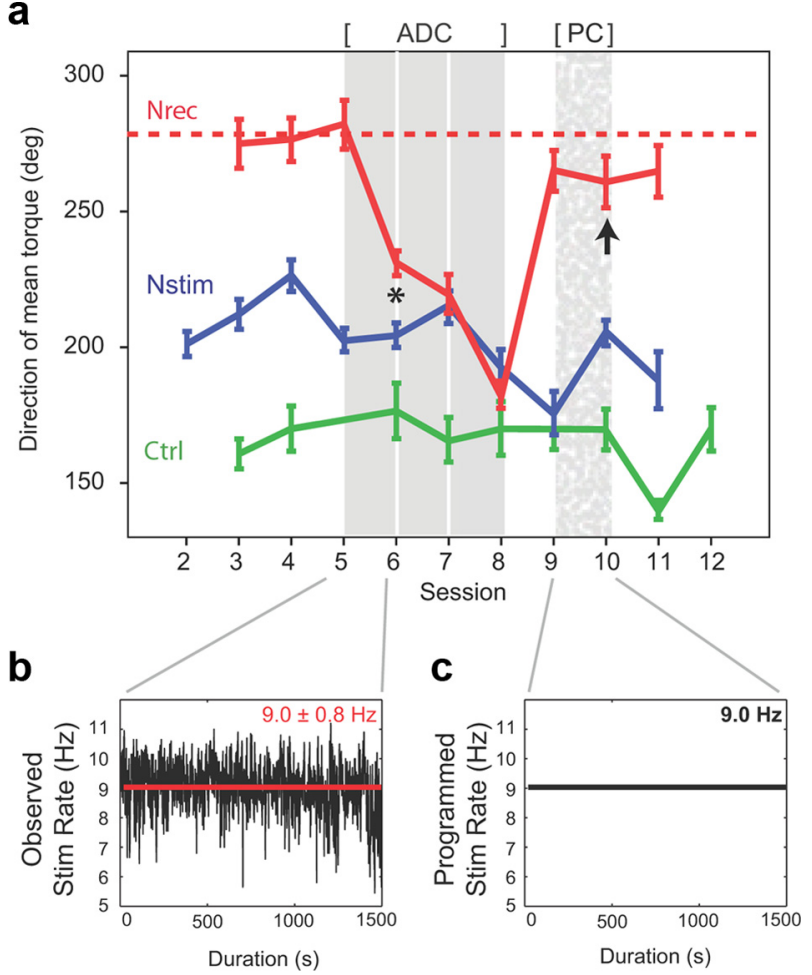

Figure 8. $A D C$ is necessary and sufficient to induce reorganization. $\boldsymbol{a}$, Direction of mean torques in separate conditioning experiment. Conditioning for 25 min produced a $46.7^{\circ} \mathrm{shift}$ in Nrec torque responses $\left({ }^{*}\right)$. The mean conditioning effect across three conditioning sessions (ADC) in block 1 was $67.1^{\circ}$. Torque responses returned to near-baseline levels (dashed red line) following termination of artificial feedback. In the second block Nrec underwent pseudoconditioning $(P C)$ using identical stimulation parameters as in *, including the behavioral condition, conditioning duration ( $25 \mathrm{~min}$ ), current intensity (44 $\mu \mathrm{A} ; 49 \%$ of movement threshold), and mean frequency $(9 \mathrm{~Hz})$. The total number of stimuli administered during $A D C$ and $P C$ was nearly identical $\left(1.39 \times 10^{4}\right.$ and $1.35 \times 10^{4}$, resp.). No reorganization was observed during $P C$ (arrow). All sessions were $24 \mathrm{~h}$ in duration, except as noted above. $\boldsymbol{b}$, The observed stimulation rate during the initial conditioning session of block 1 was $9.0 \pm 0.8 \mathrm{~Hz}$. c, The programmed stimulation rate during $P C$ was $9.0 \mathrm{~Hz}$.

\section{Summary of results}

In sum, we performed 22 conditioning sessions in four animals over a 24 month period. ADC, triggered by forelimb muscle activity, produced significant reorganization of M1 output effects in five of five experiments (100\%) and 21 of 22 (95\%) sessions. The conditioning effect was site specific: it was observed solely at Nrec sites, with no significant effect observed at Nstim or neighboring Ctrl sites (Fig. 10). The direction of mean torques evoked from Nrec shifted an average of $74.2 \pm 26.1^{\circ}$ from baseline during ADC (ANOVA, $F_{(3,48)}=49.436, p<0.001$ ), compared with $14.1 \pm 10.5^{\circ}$ for Ctrl (ANOVA, $F_{(3,32)}=2.071, p=$ n.s.) and $17.2 \pm 15.7^{\circ}$ for Nstim (ANOVA, $F_{(3,50)}=2.06, p=$ n.s.). The conditioning effect was input specific: it was observed only after stimulation synchronized to relevant muscle activity, but not after pseudoconditioning, which lacked a temporal relationship with muscle activity (Fig. 10a, red bars). Data are summarized in Table 1.

The Nrec torque vectors evoked by ICMS converged on Nstim baseline vectors during ADC, as reflected by a reduction in the angles separating Nrec and Nstim torques (Fig. 10b). Points lying below the dashed line of identity represent ICMS effects that shifted toward Nstim. All Nrec responses met this criterion (Fig. $10 b, \diamond)$. The mean angular separation between Nrec and Nstim was reduced after ADC across experiments: Nrec torques shifted toward Nstim after conditioning $(t=6.825, p<0.001$; Fig. $10 c)$, while Ctrl effects showed no statistically significant change from baseline $(t=1.481, p=$ n.s.). Normalizing these torque shifts against the separation of torque directions at baseline reveals that Nrec torques shifted $49.8 \%$ of the baseline angular separation between Nstim and Nrec, whereas Ctrl torques shifted $29.8 \%$ of the baseline angular separation between Nstim and Ctrl.

\section{Discussion}

This investigation sought to induce targeted reorganization of the motor cortex using crossed myo-cortical feedback. EMG signals from contralateral forelimb muscles triggered stimulation of M1 sites associated with different muscles. We found that ADC was necessary and sufficient to alter motor cortex output effects. Myo-cortical feedback induced rapid, robust, and input-specific reorganization. Reorganization was evident within $25 \mathrm{~min}$ of $\mathrm{ADC}$, and was sustained with intermittent conditioning for up to $5 \mathrm{~d}$. Reorganization was site specific (occurring only for the Nrec site) and input specific (occurring only with ADC). Pseudoconditioning, matched on most stimulation parameters to ADC, failed to induce reorganization. Stimulation triggered from EMG of the Mrec muscles did not produce significant conditioning effects from the Ctrl sites.

A previous study using a similar intracortical recurrent conditioning paradigm found that long-term plasticity could be produced by ADC triggered from action potentials (APs) of a single cortical unit recorded at an Nrec site (Jackson et al., 2006a). The present investigation documents that muscle activity can be an effective surrogate of associated cortical activity for inducing targeted reorganization of motor cortex. This implies that the timing of activity in Mrec is sufficiently correlated to neural activity at the Nrec site to mediate conditioning. In the study of Jackson et al. (2006a), each spike at Nrec triggered a stimulus at Nstim. In contrast, myo-cortical conditioning established a probabilistic association between Nstim and Nrec cortical activity. In the previous study reorganization of motor output effects increased over several days of conditioning and the changes in motor output effects persisted for up to $10 \mathrm{~d}$ following the end of intracortical conditioning. Employing an EMG trigger changes the neuronal elements that could be involved in conditioning, because disparate populations of corticospinal neurons are known to contribute to muscle activity. While the stimulus intensity was held constant for each conditioning experiment of Jackson et al. (2006a), we were able to investigate the intensity and stimulation ratio of conditioning stimuli delivered to Nstim as independent variables. Differences in reorganization produced by intracortical and myo-cortical conditioning may be related to differences in the neural circuits involved, the precision of the synchrony between neural elements, and the duration and intensity of this synchrony.

This investigation also introduced pseudoconditioning as a control to determine whether stimulation alone was associated with changes in motor output effects. Pseudoconditioning was identical to ADC with respect to the cortical stimulation site, stimulation parameters, and behavioral task, with the critical exception that it lacked the time-locked, phasic relationship to the activity of the forelimb muscles. Ideally, pseudoconditioning would include phasic activity to more closely mimic ADC; however, limitations in the computational capability of the neurochip precluded complex stimulus playback. The absence of significant reorganization during pseudoconditioning underscores the importance of ADC in inducing reorganization of output effects. These findings are consistent with recent evidence that 
movement-triggered vagal nerve stimulation induces reorganization of representational maps of forelimb M1 in the rodent, while neither vagal nerve stimulation nor forelimb movements alone were associated with M1 plasticity (Porter et al., 2012). The fact that the Ctrl sites showed no significant changes in output effects despite the delivery of phasic EMGtriggered stimulation confirms that phasic stimulation alone, without a temporal contingency between Ctrl and Nstim activity, was insufficient. Nrec sites demonstrated robust changes in torque vectors during conditioning relative to Ctrl and Nstim sites, which demonstrated no significant changes from baseline fluctuations in torque output. We conclude that both close temporal association (Jackson et al., 2006a) and behavioral relevance (Jenkins et al., 1990b; Nudo et al., 1996; Elbert and Rockstroh, 2004) are critical to inducing cortical plasticity in the sensorimotor system.

Accompanying the differences in methodology and design of the two studies, we observed differences in output effects and reorganization. Generally speaking, ICMSevoked torque responses were found to be more variable than in the Jackson study (2006a). In many instances, there were small fluctuations in torque direction evoked by ICMS at a given microwire site between sessions. These fluctuations increased at all sites during conditioning. In addition to these fluctuations, Ctrl sites with torque vectors nearly opposite to Nstim vectors demonstrated small shifts in torque vectors during conditioning. This may be related to activation of partially overlapping muscle fields of cortical projects during conditioning triggered by the Nrec muscle.

Movement thresholds evoked by Nstim remained largely unchanged during conditioning. In four of five experiments, no changes were observed. One experiment showed an increase in mean normalized movement thresholds similar to increases noted by Jackson et al. (2006a). Jackson et al. (2006a) observed that in some cases Nstim output effects appeared to be suppressed following conditioning, but returned over the next few days. These findings were accompanied by variability in the magnitude of Nstim output effects, although the torque directions remained stable. This variability may be attributed to long periods of repeated stimulation that may temporarily affect electrode characteristics, reduce tissue excitability (McCreery et al., 1986), or increase local inhibition. Local changes at Nstim, however, cannot account for the reorganization of output effects of Nrec observed during ADC.

The results can be explained by the potentiation of connections between Nrec and Nstim within the cortex (Hess and Donoghue, 1994; Hess et al., 1996; Rioult-Pedotti et al., 1998).

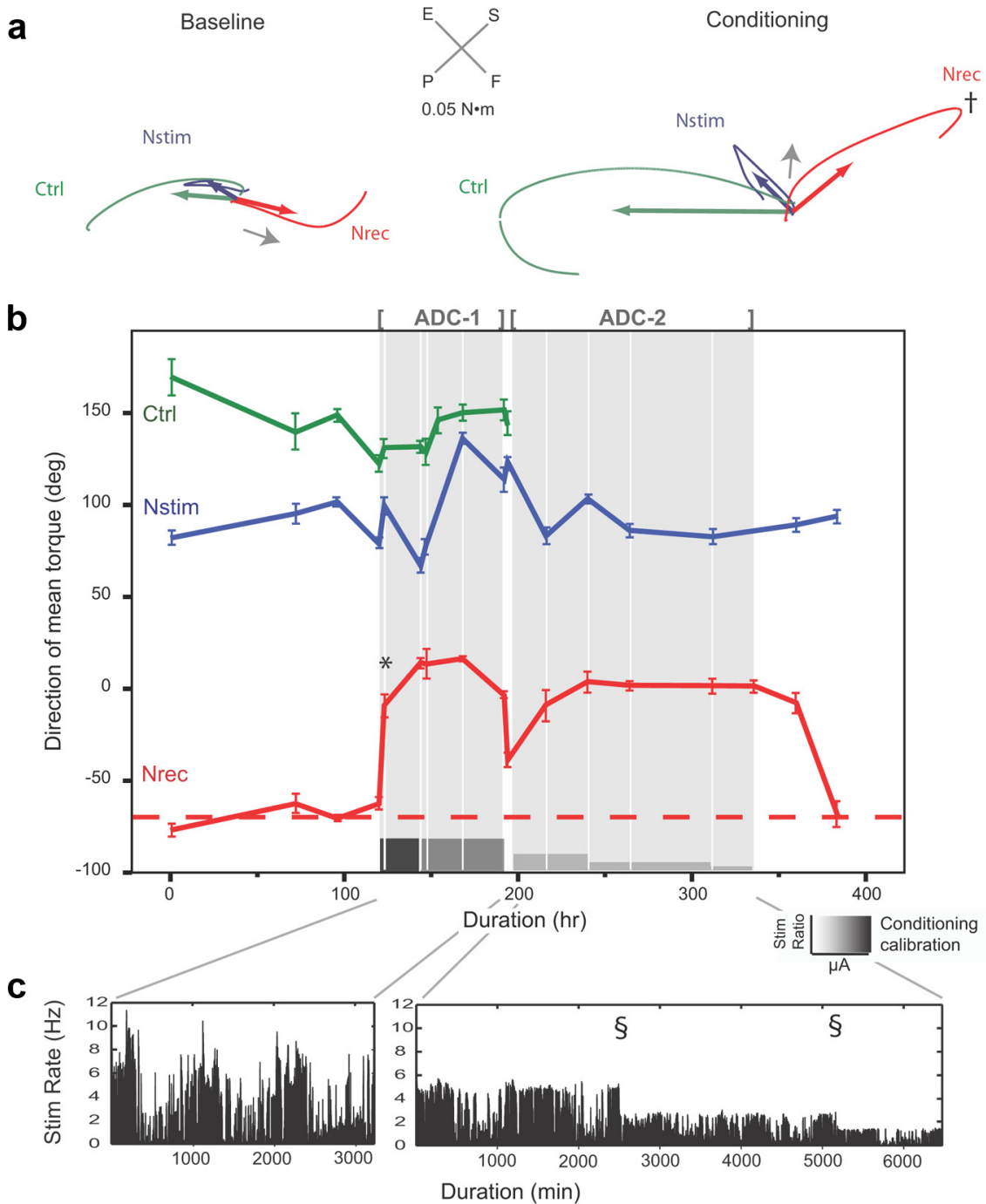

Figure 9. Sustaining plasticity by conditioning anatomical antagonists with intermittent reinforcement. $\boldsymbol{a}$, Torque responses at baseline and following $3.2 \mathrm{~h}$ of conditioning in animal Z. Nrec mean torque vectors (red arrows) shifted $59^{\circ}$ above baseline. This shift explained by significant change in the initial segment of the torque trajectories (gray arrows), and reversal of the direction forque trajectory at its inflection point ( $\dagger$ ). Data represent first conditioning session shown in $\left.b{ }^{*}\right)$. $\boldsymbol{b}$, Direction of mean torque

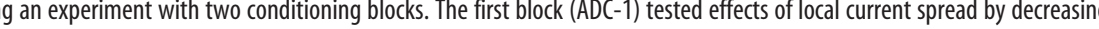
introducing intermittent reinforcement with a decreasing reinforcement schedule. The Nstim stimulation ratio (indi列 constant $(18 \mu \mathrm{A})$. Intermittent reinforcement maintained cortical reorganization throughout block 2 . Nrec baseline torque shown by dashed red line. Ctrl microwire was lost to mechanical breakage between blocks. c, Stimulation rates during conditioning block 1 (left) and 2 (right). Stimulation rates were highly phasic, reflecting the animal's unrestrained limb movements. During block 2 , the stimulation rate was reduced in two steps $(\S)$ by increasing the ratio of EMG triggers-to-intracortical stimuli during conditioning. Rate data plotted for 1 min bins.

We hypothesize that weak synaptic connections between cortical sites, or their downstream projections, are strengthened by the arrival of normal presynaptic volleys at Nstim coincident with induced postsynaptic depolarizations produced by movementrelated stimulation (Fig. 11). Subcortical sites where this conditioning paradigm could induce changes are illustrated by the "interneuron" pathway in Figure 11d. Additional sites of convergence, not illustrated, include the cerebellum. After conditioning, ICMS at Nrec recruits pathways normally activated from Nstim, resulting in a shift in evoked wrist torques toward those evoked from Nstim. Further, ADC strengthens these connections to the point that intermittent reinforcement alone is sufficient to maintain them over multiple days. In this context, the intermittent 
a
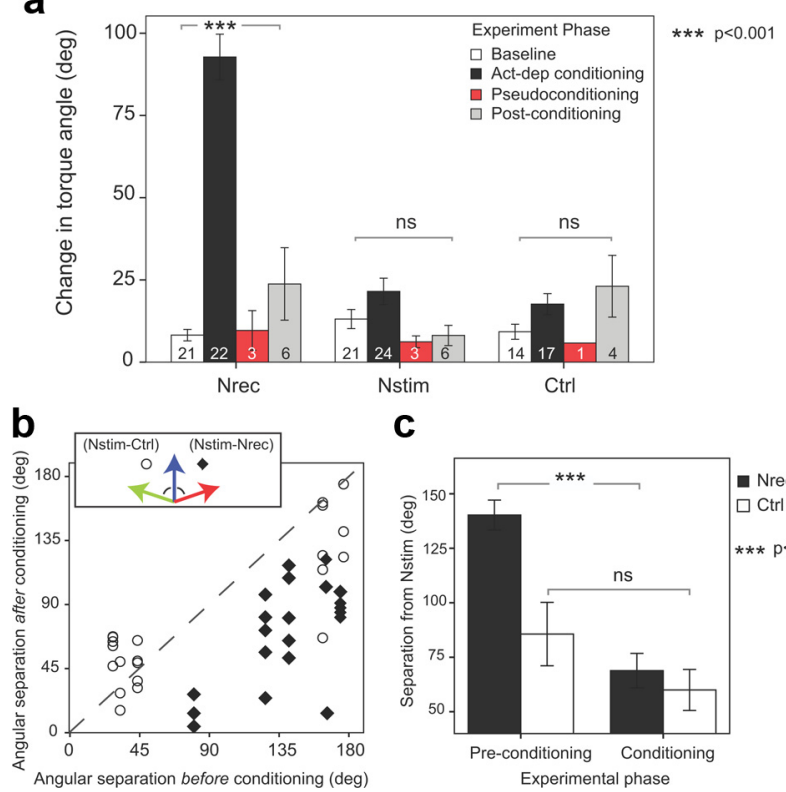

C

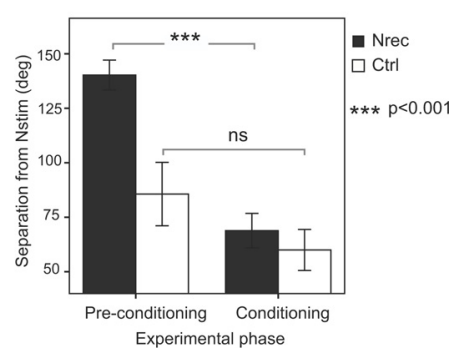

Figure 10. Reorganization is site specific and input specific. $\boldsymbol{a}$, Change in mean torque direction for each cortical site for all stages of the experiments. ADC (black bars) produced significant reorganization of torque output at Nrec, but not Nstim or Ctrl sites. Pseudoconditioning (red bars) failed to induce reorganization at any cortical site. Pooled data from all experiments in four animals; number of observations shown in each bar. Baseline variability in torque output shown in white. Data represent mean \pm SEM. ${ }^{* *} p<0.001$, ns, not significant. $\boldsymbol{b}$, Summary of $A D C$ results illustrated by the angular separation of Nrec and Ctrl ICMS effects from the direction of Nstim mean torque (inset). Nrec ICMS effects ( ) fall below the dashed line of identity in every instance, indicating a consistent shift toward Nstim after conditioning. c, Baseline and conditioned mean ICMS effects for Nrec (filled bars) and (trl (open bars). Conditioning resulted in reduced separation of torque responses between Nrec and Nstim ${ }^{* * *} p<0.001$, independent samples $t$ test, $t=6.825$ ). There were no significant differences between baseline and conditioning Ctrl means.

stimulation "reinforces" the newly established conditioning effect, despite the decreased probability of conditioning stimulation relative to EMG triggers.

Accumulating experimental evidence indicates that motor cortex plasticity involves mechanisms of STDP. The studies of Jackson et al. (2006a) and Rebesco et al. (2010) demonstrated that effective spike-stimulus delays occurred within the $50 \mathrm{~ms}$ of STDP effects. Given the nature of the EMG signal we did not test the effects of varying delays. However, our results do demonstrate that the relation between cells at cortical site Nrec and muscle activity in Mrec is sufficiently tight to induce plasticity within primate motor cortex or other effective sites.

Horizontal connections within M1 are capable of synaptic modification, including LTP (Hess and Donoghue, 1994, 1996, 1999). Motor cortex LTP requires activation of NMDA receptors (Hess et al., 1996) and dendritic $\mathrm{Ca}^{2+}$ influx (Hess, 2002) as a principal mechanism of STDP. Coincident postsynaptic APs and unitary EPSPs result in increased amplitude of subsequent EPSPs (Markram et al., 1997). Blocking NMDA receptors prevents plastic changes in EPSPs. The mechanisms that underlie the plastic changes observed here are likely to be the same as those that underlie the changes observed following altered physiological circumstances (Pascual-Leone et al., 1995; Nudo and Milliken, 1996; Nudo et al., 1996; Rioult-Pedotti et al., 2000; Sanes and Donoghue, 2000; Jackson et al., 2006a). In vitro (Markram et al., 1997) and in vivo (Jackson et al., 2006a; Rebesco et al., 2010; Thabit et al., 2010) investigations of cortical plasticity support this hypothesis and are consistent with STDP. Additional inves- tigation into the cellular mechanism of synaptic strengthening following ADC is warranted. Convergent sites of synaptic strengthening at subcortical, cerebellar, or spinal levels are all consistent with the present data (Fig. 11d, red boutons).

The fact that the conditioned changes are extinguished in the presence of normal spontaneous activity without intermittent reinforcement is also consistent with the role of STDP mechanisms. Conditioning protocols that induce cellular changes are typically reversed within minutes to hours by normal spiking activity (Frégnac et al., 1988; Ahissar et al., 1992; Hess and Donoghue, 1999; Mu and Poo, 2006). Representational borders begin to return to their baseline topology within minutes following stimulation-induced expansion of cortical movement representations (Nudo et al., 1990). Functional changes in muscle contraction force and EMG following conditioning fade within $30 \mathrm{~min}$ of cessation of associative stimulation (Taylor and Martin, 2009).

Non-STDP mechanisms could also be involved in M1 reorganization. The rapid reorganization observed in the present study outpaces axon sprouting and synaptogenesis (Kleim et al., 1996), making these mechanisms unlikely candidates to explain reorganization observed within minutes. Another alternative is fluctuations in the pattern of local inhibitory and excitatory inputs, which transiently influence M1 output effects on a shorter timescale. For example, when intracortical inhibition is removed with bicuculline, a GABA antagonist, repeated stimulation unmasks extant, high-threshold excitatory connections resulting in the appearance of reorganization (Jacobs and Donoghue, 1991). Temporary release of inhibition may also facilitate synaptic strengthening between neighboring cell populations (Artola and Singer, 1987; Hess and Donoghue, 1994). Repetitive suprathreshold stimulation ( $8 \times$ threshold $)$ can induce a transient blockage of neural activity adjacent to the stimulating electrode (Asanuma and Ward, 1971). Repetitive high-frequency stimulation at lower intensities ( $2 \times$ threshold) expands movement representations in rodent motor cortex when delivered constantly for $1-3 \mathrm{~h}$ in an anesthetized preparation (Nudo et al., 1990). However, neither representational expansion nor local changes in inhibitory inputs explain the results observed here, because (1) the reorganization of ICMS output effects were observed from the Nrec site, which was not the site receiving stimulation during conditioning; (2) pseudoconditioning failed to produce reorganization despite matched stimulation parameters; and (3) evoked output effects from Nstim and Ctrl remained largely unchanged after conditioning. Finally, movement-triggered vagal nerve stimulation has recently been shown to result in expansion in M1 representational maps, with the proposed mechanism related to synergistic effect of plasticity-enhancing molecules, including acetylcholine, norepinephrine, serotonin, and brain-derived neurotropic factor (Porter et al., 2012). Future investigations targeting the neuropharmacology of activity-dependent plasticity are indicated.

The ease of changing the relationship between cortical sites and associated muscles suggests that these relations are normally maintained through physiological feedback loops. Indeed, recordings from cortical and spinal neurons in these pathways reveal very similar response patterns that would reinforce their underlying synaptic connections (Fig. 11e). Corticomotoneuronal cells (CM) have the most direct connections to spinal motoneurons (Cheney and Fetz, 1984). Motor units in forelimb muscles exhibit corresponding response patterns during motor tasks (for review, see Fetz et al., 2002a). Normal movements are associated with afferent discharge from muscle receptors that is proportional to EMG, as recorded in dorsal root ganglia (Fig. 11e, 
a

ICMS delivered separately to each cortical site activates associated muscles and produces torque responses at baseline

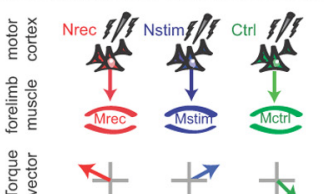

b

Activity-dependent conditioning induces synchonization between Nrec and Nstim, strengthening shared connections

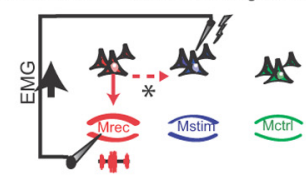

subthreshold

stimulation

* shared

connections

C Nrec ICMS activates strengthened pathways following conditioning

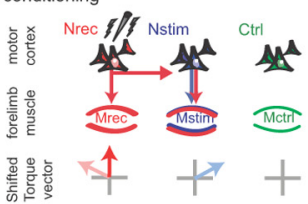

d

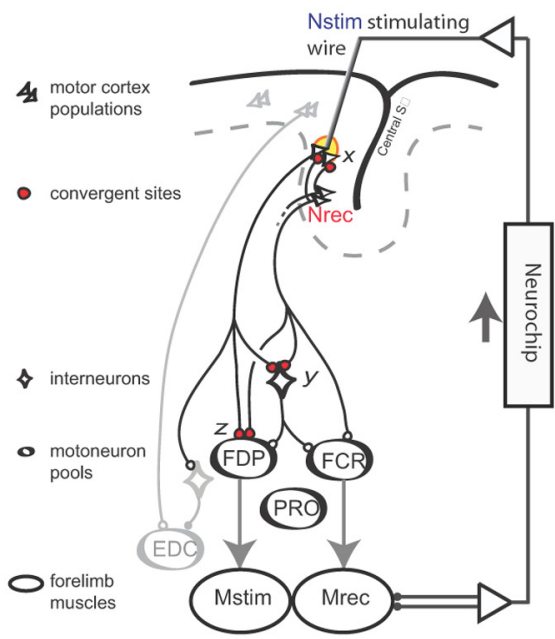

e

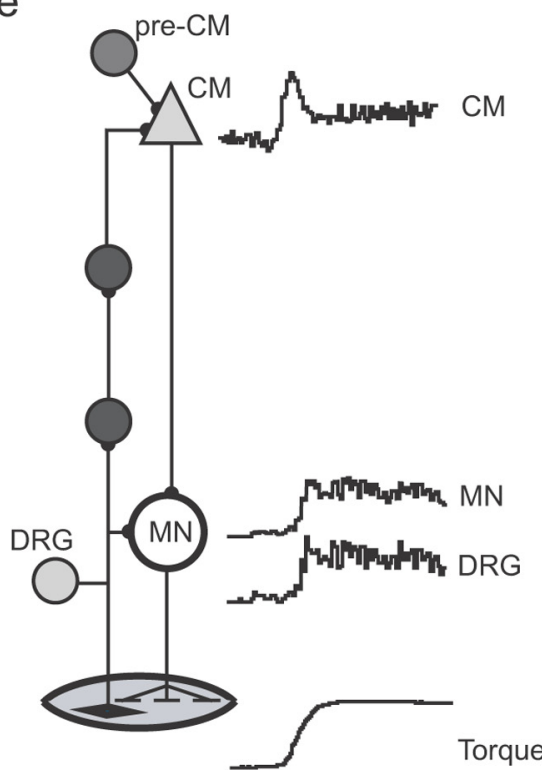

Figure 11. Proposed mechanism of reorganization. $\boldsymbol{a}$, ICMS testing defines baseline responses. $\boldsymbol{b}$, Nstim ADC evokes postsynaptic depolarization synchronized to presynaptic inputs from Nrec horizontal connections normally activity during movement (dashed red arrow). c, Following conditioning, ICMS at Nrec activates strengthened pathways to produce responses that incorporate Nstim projections. Similar mechanisms could occur at other sites of convergence. $\boldsymbol{d}$, Putative convergent sites for synaptic strengthening. The projections of separate motor cortex populations are illustrated in a hypothetical conditioning experiment pairing anatomical agonists FCR and FDP. A stimulating microwire terminates in the proximity of Nstim. ADC strengthens synapses shown in red $(" x ")$ that mediate connections from Nrec during volitional movements. A proportion of the population is activated directly by focal current injection (yellow region). Subcortical and spinal sites of convergence are illustrated by red boutons ( $y$ and $z$ ).e, Corticomuscle loop. A schematic of the minimal corticomuscle pathways (left) and response patterns of involved neurons (right). CM cells have the most direct, monosynaptic connections on spinal motoneurons (MN), although polysynaptic linkages via other corticospinal cells exist. The afferent pathway begins with la afferents whose somas are located in the DRG. The predominant response type of these cell populations recorded during a ramp-and-hold wrist movement is shown at right (Adapted from Fetz et al., 2002b). Additional inputs to the CM cell, including central cells driving the CM cell, are represented by the pre-CM cell.

DRG). This afferent activity also activates CM cells (Cheney and Fetz, 1984), creating a recurrent corticomuscular loop. The similar response patterns indicate that elements throughout the recurrent loop are coactivated in a way that would maintain the strength of their synaptic connections. Central inputs to the CM cell, including upstream neurons driving the $\mathrm{CM}$ cell (pre-CM cells), mediate the activation of CM cells during volitional movements. Under normal conditions the consistent coactivation of the inputs from pre-CM cells and from the afferent pathway maintains their synaptic strength. During our EMG-triggered stimulation these afferents are consistently activated in synchrony with another muscle, artificially strengthening the connection from that muscle's cortical representation. Once established, intermittent activation is able to maintain input specificity. However, after termination of the artificial activation the conditioning effects are reversed by normal reciprocal connections.

The conditioning effects would be expected to appear in cortically evoked muscle activity as well as changes in torque outputs. However, train-triggered EMG responses from sampled muscles were more variable than wrist torque responses, which represent the net sum of forces exerted by wrist and hand muscles. The variability in muscle responses may reflect physiological factors that could not be controlled directly. First, these experiments paired muscles with different actions (e.g., FDS, EDC, supinator) in an attempt to test the range of conditionable connections. Muscles chosen to trigger recurrent conditioning may fall within the muscle field of precentral cells that have excitatory and/or inhibitory influences upon motoneuron pools with agonist or antagonist actions relative to the trigger muscle. This intrinsic connectivity complicates predictions about the expected EMG changes in response to conditioning. Torque responses capture the actions of all wrist muscles, and therefore serve as a more comprehensive measure of motor output changes than a sampling of forelimb muscles. Second, muscle responses are influenced by the state of excitation of the $\alpha$-motoneurons, which are affected by various descending motor systems including the corticospinal, rubrospinal, vestibulospinal, and reticulospinal pathways (Lemon, 2008), as well as spinal reflex pathways and muscle afferents (Komori et al., 1992). The net activational state of motoneuronal pools and corticospinal circuitry during a resting task is difficult to control given the absence of volitional behavior. While we carefully omitted trials when subjects actively moved or failed to attend to the task, it was not possible to prevent the subjects from occasionally moving or shifting attention between trials. Changes in wrist position between trials may affect the excitability of motoneurons by changing the muscle thresholds. Changes in corticospinal excitability, which accompany wrist threshold position resetting, are independent of changes in EMG activity (Raptis et al., 2010). The degree to which changes in corticospinal excitability contributes to the variability of muscle responses during ICMS at rest warrants future study.

A second factor that potentially influenced the variability of EMG responses is the fact that the EMG electrodes were applied transcutaneously for each recording session. The implanted muscles were identified by intramuscular stimulation, which is ultimately mediated by the stimulated nerves. Although this was similar for each session, the motor units recorded could have been sufficiently different to account for the variation in evoked responses.

Directing neocortical plasticity through recurrent activitydependent stimulation has clinical implications for effective treatment of stroke and brain injury through plasticity or neural prostheses. Targeted plasticity has been proposed as a therapeutic 
method of reversing pathological neural activity (Engineer et al., 2011). Because the reorganization observed here required intermittent stimulation, clinical application of targeted reorganization based on these findings would probably require chronic stimulation to maintain efficacy. Similar approaches are commonly used in neurological patients, as in the case of deep brain stimulation (Lyons, 2011), vagal nerve stimulation (Privitera et al., 2002), spinal cord stimulation (Compton et al., 2012), and motor cortex stimulation (Compton et al., 2012). In each of these treatments, chronic stimulation is required to maintain efficacy. Chronic centrally triggered brain stimulation is under investigation as a treatment for seizure disorders. The Neuropace RNS system, for instance, records electrocorticography in real-time and delivers recurrent stimulation "to normalize" brain activity when seizure activity detected (Morrell and Investigators, 2009). A novel divergence from these approaches would be the application of activity-dependent recurrent stimulation triggered by peripheral physiological signals. Employment of peripherally triggered recurrent stimulation offers the potential advantage of not requiring stable chronic neural recordings. Additional advantages include calibrated volitional control of effects and potential strengthening of weakened corticomuscular linkages. Peripherally triggered activity-dependent stimulation could employ the same implantable battery powered platforms currently used for deep brain stimulation. Additional potential advantages of using EMG activity over cortical activity as a source for inducing targeted reorganization in clinical brain-computer interface applications are that EMG activity is (1) readily accessible, (2) has a favorable signal-to-noise ratio, and (3) may be recorded indefinitely without the need of invasive procedures.

It is also possible that more prolonged conditioning would result in more prolonged structural changes in synaptic connections (Cramer et al., 2011). Another strategy to promote longer lasting effects would be to introduce additional mechanisms to promote effective long-lasting plasticity, like cortical DC polarization or concurrent delivery of neuromodulators. These possibilities would be issues for further investigation.

\section{References}

Ahissar E, Vaadia E, Ahissar M, Bergman H, Arieli A, Abeles M (1992) Dependence of cortical plasticity on correlated activity of single neurons and on behavioral context. Science 257:1412-1415. CrossRef Medline

Artola A, Singer W (1987) Long-term potentiation and NMDA receptors in rat visual cortex. Nature 330:649-652. CrossRef Medline

Asanuma H, Rosén I (1972) Topographical organization of cortical efferent zones projecting to distal forelimb muscles in the monkey. Exp Brain Res 14:243-256. CrossRef Medline

Asanuma H, Ward JE (1971) Patterns of contraction of distal forelimb muscles produced by intracortical stimulation in cats. Brain Res 27:97-109. CrossRef Medline

Cheney PD, Fetz EE (1984) Corticomotoneuronal cells contribute to longlatency stretch reflexes in the rhesus monkey. J Physiol 349:249-272. Medline

Compton AK, Shah B, Hayek SM (2012) Spinal cord stimulation: a review. Curr Pain Headache Rep 16:35-42. CrossRef Medline

Cramer SC, Sur M, Dobkin BH, O'Brien C, Sanger TD, Trojanowski JQ, Rumsey JM, Hicks R, Cameron J, Chen D, Chen WG, Cohen LG, deCharms C, Duffy CJ, Eden GF, Fetz EE, Filart R, Freund M, Grant SJ, Haber S, et al. (2011) Harnessing neuroplasticity for clinical applications. Brain 134:1591-1609. CrossRef Medline

Donoghue JP, Sanes JN (1987) Peripheral nerve injury in developing rats reorganizes representation pattern in motor cortex. Proc Natl Acad Sci U S A 84:1123-1126. CrossRef Medline

Elbert T, Rockstroh B (2004) Reorganization of human cerebral cortex: the range of changes following use and injury. Neuroscientist 10:129-141. CrossRef Medline

Elbert T, Flor H, Birbaumer N, Knecht S, Hampson S, Larbig W, Taub E
(1994) Extensive reorganization of the somatosensory cortex in adult humans after nervous system injury. Neuroreport 5:2593-2597. CrossRef Medline

Engineer ND, Riley JR, Seale JD, Vrana WA, Shetake JA, Sudanagunta SP, Borland MS, Kilgard MP (2011) Reversing pathological neural activity using targeted plasticity. Nature 470:101-104. CrossRef Medline

Fetz EE, Perlmutter SI, Prut Y, Seki K (2002a) Functional properties of primate spinal interneurones during voluntary hand movements. Adv Exp Med Biol 508:265-271. CrossRef Medline

Fetz EE, Perlmutter SI, Prut Y, Seki K, Votaw S (2002b) Roles of primate spinal interneurons in preparation and execution of voluntary hand movement. Brain Res Brain Res Rev 40:53-65. CrossRef Medline

Frégnac Y, Shulz D, Thorpe S, Bienenstock E (1988) A cellular analogue of visual cortical plasticity. Nature 333:367-370. CrossRef Medline

Freund P, Rothwell J, Craggs M, Thompson AJ, Bestmann S (2011) Corticomotor representation to the human forearm muscle changes following cervical spinal cord injury. Eur J Neurosci 34:1839-1846. CrossRef Medline

Hebb DO (1949) The organization of behavior: a neuropsychological theory. New York: Wiley.

Hess G (2002) Calcium-induced long-term potentiation in horizontal connections of rat motor cortex. Brain Res 952:142-145. CrossRef Medline

Hess G, Donoghue JP (1994) Long-term potentiation of horizontal connections provides a mechanism to reorganize cortical motor maps. J Neurophysiol 71:2543-2547. Medline

Hess G, Donoghue JP (1996) Long-term potentiation and long-term depression of horizontal connections in rat motor cortex. Acta Neurobiol Exp 56:397-405. Medline

Hess G, Donoghue JP (1999) Facilitation of long-term potentiation in layer II/III horizontal connections of rat motor cortex following layer I stimulation: route of effect and cholinergic contributions. Exp Brain Res 127: 279-290. CrossRef Medline

Hess G, Aizenman CD, Donoghue JP (1996) Conditions for the induction of long-term potentiation in layer II/III horizontal connections of the rat motor cortex. J Neurophysiol 75:1765-1778. Medline

Hikosaka O, Rand MK, Nakamura K, Miyachi S, Kitaguchi K, Sakai K, Lu X, Shimo Y (2002) Long-term retention of motor skill in macaque monkeys and humans. Exp Brain Res 147:494-504. CrossRef Medline

Jackson A, Fetz EE (2007) Compact movable microwire array for long-term chronic unit recording in cerebral cortex of primates. J Neurophysiol 98:3109-3118. CrossRef Medline

Jackson A, Mavoori J, Fetz EE (2006a) Long-term motor cortex plasticity induced by an electronic neural implant. Nature 444:56-60. CrossRef Medline

Jackson A, Moritz CT, Mavoori J, Lucas TH, Fetz EE (2006b) The Neurochip BCI: towards a neural prosthesis for upper limb function. IEEE Trans Neural Syst Rehabil Eng 14:187-190. CrossRef Medline

Jacobs KM, Donoghue JP (1991) Reshaping the cortical motor map by unmasking latent intracortical connections. Science 251:944-947. CrossRef Medline

Jankowska E, Padel Y, Tanaka R (1975) The mode of activation of pyramidal tract cells by intracortical stimuli. J Physiol 249:617-636. Medline

Jenkins WM, Merzenich MM, Recanzone G (1990a) Neocortical representational dynamics in adult primates: implications for neuropsychology. Neuropsychologia 28:573-584. CrossRef Medline

Jenkins WM, Merzenich MM, Ochs MT, Allard T, Guic-Robles E (1990b) Functional reorganization of somatosensory representations within area $3 \mathrm{~b}$ of adult owl monkey after behaviorally controlled tactile stimulation. J Neurophysiol 63:82-104. Medline

Kaas JH, Merzenich MM, Killackey HP (1983) The reorganization of somatosensory cortex following peripheral nerve damage in adult and developing mammals. Annu Rev Neurosci 6:325-356. CrossRef Medline

Kleim JA, Lussnig E, Schwarz ER, Comery TA, Greenough WT (1996) Synaptogenesis and fos expression in the motor cortex of the adult rat after complex motor skill acquisition. J Neurosci 16:4529-4535. Medline

Komori T, Watson BV, Brown WF (1992) Influence of peripheral afferents on cortical and spinal motoneuron excitability. Muscle Nerve 15:48-51. CrossRef Medline

Lemon RN (2008) Descending pathways in motor control. Annu Rev Neurosci 31:195-218. CrossRef Medline

Lyons MK (2011) Deep brain stimulation: current and future clinical applications. Mayo Clin Proc 86:662-672. CrossRef Medline 
Markram H, Lübke J, Frotscher M, Sakmann B (1997) Regulation of synaptic efficacy by coincidence of postsynaptic APs and EPSPs. Science 275: 213-215. CrossRef Medline

Mavoori J, Jackson A, Diorio C, Fetz E (2005) An autonomous implantable computer for neural recording and stimulation in unrestrained primates. J Neurosci Methods 148:71-77. CrossRef Medline

McCreery DB, Bullara LA, Agnew WF (1986) Neuronal activity evoked by chronically implanted intracortical microelectrodes. Exp Neurol 92:147161. CrossRef Medline

Morrell MJ, Investigators RP (2009) Results of a multicenter double blinded randomized controlled pivotal investigation of the RNS system for treatment of intractable partial epilepsy in adults. Presented at the $63^{\text {rd }}$ Annual American Epilepsy Society Meeting, Boston, MA, December 4-8.

Mu Y, Poo MM (2006) Spike time-dependent LTP/LTD mediates visual experience-dependent plasticity in a developing retinotectal system. Neuron 50:115-125. CrossRef Medline

Nudo RJ, Milliken GW (1996) Reorganization of movement representations in primary motor cortex following focal ischemic infarcts. J Neurophysiol 75:2144-2149. Medline

Nudo RJ, Jenkins WM, Merzenich MM (1990) Repetitive microstimulation alters the cortical representation of movements in adult rats. Somatosens Mot Res 7:463-483. CrossRef Medline

Nudo RJ, Milliken GW, Jenkins WM, Merzenich MM (1996) Usedependent alterations of movement representations in primary motor cortex of adult squirrel monkeys. J Neurosci 16:785-807. Medline

Pascual-Leone A, Grafman J, Hallett M (1994) Modulation of cortical motor output maps during development of implicit and explicit knowledge. Science 263:1287-1289. CrossRef Medline

Pascual-Leone A, Nguyet D, Cohen LG, Brasil-Neto JP, Cammarota A, Hallett M (1995) Modulation of muscle responses evoked by transcranial magnetic stimulation during the acquisition of new fine motor skills. J Neurophysiol 74:1037-1045. Medline

Pons TP, Garraghty PE, Ommaya AK, Kaas JH, Taub E, Mishkin M (1991) Massive cortical reorganization after sensory deafferentation in adult macaques. Science 252:1857-1860. CrossRef Medline

Porter BA, Khodaparast N, Fayyaz T, Cheung RJ, Ahmed SS, Vrana WA, Rennaker RL 2nd, Kilgard MP (2012) Repeatedly pairing vagus nerve stimulation with a movement reorganizes primary motor cortex. Cereb Cortex 22:2365-2374. CrossRef Medline

Privitera MD, Welty TE, Ficker DM, Welge J (2002) Vagus nerve stimulation for partial seizures. Cochrane Database Syst Rev CD002896.

Qi HX, Stepniewska I, Kaas JH (2000) Reorganization of primary motor cortex in adult macaque monkeys with long-standing amputations. J Neurophysiol 84:2133-2147. Medline

Raptis H, Burtet L, Forget R, Feldman AG (2010) Control of wrist position and muscle relaxation by shifting spatial frames of reference for motoneuronal recruitment: possible involvement of corticospinal pathways. J Physiol 588:1551-1570. CrossRef Medline

Rebesco JM, Stevenson IH, Kording KP, Solla SA, Miller LE (2010) Rewiring neural interactions by micro-stimulation. Front Syst Neurosci 4.

Recanzone GH, Merzenich MM, Jenkins WM (1992a) Frequency discrimination training engaging a restricted skin surface results in an emergence of a cutaneous response zone in cortical area 3a. J Neurophysiol 67:10571070. Medline

Recanzone GH, Merzenich MM, Jenkins WM, Grajski KA, Dinse HR (1992b) Topographic reorganization of the hand representation in cortical area $3 \mathrm{~b}$ of owl monkeys trained in a frequency-discrimination task. J Neurophysiol 67:1031-1056. Medline

Rioult-Pedotti MS, Friedman D, Hess G, Donoghue JP (1998) Strengthening of horizontal cortical connections following skill learning. Nat Neurosci 1:230-234. CrossRef Medline

Rioult-Pedotti MS, Friedman D, Donoghue JP (2000) Learning-induced LTP in neocortex. Science 290:533-536. CrossRef Medline

Rosén I, Asanuma H (1972) Peripheral afferent inputs to the forelimb area of the monkey motor cortex: input-output relations. Exp Brain Res 14: 257-273. CrossRef Medline

Sanes JN, Donoghue JP (2000) Plasticity and primary motor cortex. Annu Rev Neurosci 23:393-415. CrossRef Medline

Sanes JN, Suner S, Lando JF, Donoghue JP (1988) Rapid reorganization of adult rat motor cortex somatic representation patterns after motor nerve injury. Proc Natl Acad Sci U S A 85:2003-2007. CrossRef Medline

Stoney SD Jr, Thompson WD, Asanuma H (1968) Excitation of pyramidal tract cells by intracortical microstimulation: effective extent of stimulating current. J Neurophysiol 31:659-669. Medline

Taylor JL, Martin PG (2009) Voluntary motor output is altered by spiketiming-dependent changes in the human corticospinal pathway. J Neurosci 29:11708-11716. CrossRef Medline

Thabit MN, Ueki Y, Koganemaru S, Fawi G, Fukuyama H, Mima T (2010) Movement-related cortical stimulation can induce human motor plasticity. J Neurosci 30:11529-11536. CrossRef Medline

Wang H, Wang X, Scheich H (1996) LTD and LTP induced by transcranial magnetic stimulation in auditory cortex. Neuroreport 7:521-525. CrossRef Medline 